\title{
Non-parametric seasonal unit root tests under periodic non-stationary volatility
}

\author{
Kemal Çag̃lar Gög̃ebakan ${ }^{1,2}$ (D) Burak Alparslan Eroglu 3
}

Received: 11 June 2021 / Accepted: 7 February 2022 / Published online: 7 March 2022

(c) The Author(s), under exclusive licence to Springer-Verlag GmbH Germany, part of Springer Nature 2022

\begin{abstract}
This paper presents a new non-parametric seasonal unit root testing framework that is robust to periodic non-stationary volatility in innovation variance by making an extension to the fractional seasonal variance ratio unit root tests of Eroğlu et al. (Econ Lett 167:75-80,2018). The setup allows for both periodic heteroskedasticity structure of Burridge and Taylar (J Econ 104(1):91-117, 2001) and non-stationary volatility structure of Cavaliere and Taylor (Econ Theory 24(1):43-71, 2008). We show that the limiting null distributions of the variance ratio tests depend on nuisance parameters derived from the underlying volatility process. Monte Carlo simulations show that the standard variance ratio tests can be substantially oversized in the presence of such effects. Consequently, we propose wild bootstrap implementations of the variance ratio tests. Wild bootstrap resampling schemes are shown to deliver asymptotically pivotal inference. The simulation evidence depicts that the proposed bootstrap tests perform well in practice and essentially correct the size problems observed in the standard fractional seasonal variance ratio tests, even under extreme patterns of heteroskedasticity.
\end{abstract}

Keywords Seasonal unit root · Non-stationary volatility · Variance ratio $\cdot$ Wild bootstrap

Kemal Çag̃lar Gög̃ebakan gogebaka@ohsu.edu

Burak Alparslan Eroglu burak.eroglu@bilgi.edu.tr

1 Cancer Early Detection Advanced Research Center, Knight Cancer Institute, Oregon Health and Science University, Portland, USA

2 Knight Cancer Institute, Oregon Health and Science University, Portland, USA

3 Department of Economics, Eski Silahtarag̃a Elektrik Santrali, İstanbul Bilgi University, Kazım Karabekir Cad, No: 2/13, L1 203, 34060 Eyüp, İstanbul, Turkey

4 Department of Economics, İstanbul Bilgi University, İstanbul, Turkey 


\section{Introduction}

There is a growing body of the literature related to the heteroscedasticity patterns in seasonal economic and financial variables. These patterns can be grouped into two categories. The first is periodic heteroscedasticity (hereafter $\mathrm{PH}$ ), where shocks in some seasons are more variable than others. It is documented that there is significant evidence of PH in variables such as manufacturing series, marginal cost curves, unemployment rates (see Proietti 1998; Franses et al. 1996; Beaulieu and Miron 1993). The second one is non-stationary volatility, where there are permanent changes in volatility within years. Some variables which exhibit non-stationary volatility are energy consumption, core CPI, real GDP growth and exchange rates (see McConnell and Perez-Quiros 2000; Kahn et al. 2002; Van Dijk et al. 2002). The empirical evidence of non-stationary volatility coupled with seasonal non-stationarity led the researchers to investigate the seasonal unit root tests under heteroscedasticity. For instance, Burridge and Taylor (2001, 2004) and Cavaliere et al. (2017) suggest heteroscedasticity robust versions of the parametric seasonal unit tests of Hylleberg et al. (1990) (hereafter, HEGY). However, the existing non-parametric seasonal unit root tests are still based on the assumption of homoscedasticity.

In this paper, we aim to improve the performance of the non-parametric seasonal unit root tests under both $\mathrm{PH}$ and non-stationary volatility. To accomplish this improvement, we first consider the asymptotic properties of the fractional seasonal variance ratio (hereafter FSVR) test statistics of Eroğlu et al. (2018) under the types of heteroscedasticity as mentioned above. We demonstrate that the asymptotic distributions of FSVR test statistics depend on nuisance parameters arising from the heteroscedasticity present in the innovations. The limiting distributions of the joint frequency FSVR tests primarily depend on seasonal heterogeneity parameters and can not be directly simulated. Therefore, we suggest a wild bootstrap-based procedure that provides good approximations to the limiting distributions of each FSVR test statistic.

This new testing design has several advantages. First, it is fully non-parametric and does not necessitate the parametric estimation as in Burridge and Taylor (2001) and in Cavaliere et al. (2017). Second, the non-parametric wild bootstrap reduces severe size distortions in the presence of heteroscedasticity and maintains the tuning parameter-free nature of the proposed tests. Third, the wild bootstrap implemented in this paper replicates the pattern of heteroscedasticity in the seasonal innovations without estimating the variance profile. Finally, bootstrap saves us from simulating the asymptotic distributions of the test statistics of interest. This feature is important since it is impossible to simulate the test statistics' asymptotic distribution in some cases.

The remainder of the paper is organized as follows. Section 2 introduces a seasonal data generating process (DGP) with periodic non-stationary volatility and details the assumptions under which we will work. In Sect. 3, the effects of periodic non-stationary volatility on the non-parametric seasonal unit root tests are discussed. Wild bootstrap algorithm of the FSVR unit root tests are defined, and the limiting null distributions of the associated wild bootstrap FSVR tests are given in Sect. 4. The finite sample properties of the wild bootstrap HEGY and FSVR unit root tests are explored through Monte Carlo simulation in Sect. 5. This section precedes the empirical application 
of the proposed methods on the U.S. unemployment data. Finally, Sect. 7 concludes. The proofs of the theorems and lemmas are placed in Appendix A, and the tables for the simulation study and empirical exercises are given in Appendix B. Throughout the article, ${ }^{\top}$ is the transpose operator, $\lfloor x\rfloor$ indicates the largest integer less than $x$, $x:=y$ reads as " $x$ is defined as $y$ ", and $\rightarrow$ indicates the week convergence as $N$ goes to infinity.

\section{The heteteroskedastic seasonal model and assumptions}

In this section, we firstly present the univariate heteroscedastic seasonal time series $\left\{x_{S n+s}\right\}$ with the following data generating process (DGP):

$$
\begin{aligned}
\alpha^{*}(L) x_{S n+s} & =u_{S n+s}, \quad s \in\{1-S, \ldots, 0\}, \quad n \in\{1, \ldots, N\}, \\
u_{S n+s} & =\Psi(L) \varepsilon_{S n+s}, \\
\varepsilon_{S n+s} & =\sigma_{S n+s} e_{S n+s},
\end{aligned}
$$

where $S$ represents the number of seasons, $\alpha^{*}(L)=1-\sum_{j=1}^{S} \alpha_{j}^{*} L^{j}$ is an $A R(S)$ polynomial which determines the seasonal unit root. Let $\omega_{j}=2 \pi j / S$ denotes the frequency in radial. The seasonal lag polynomial can be expanded as $\alpha^{*}(L)=\prod_{j=0}^{\lfloor S / 2\rfloor} \omega_{j}(L)$ with $\omega_{0}=\left(1-\alpha_{0} L\right)$ (unit root at zero frequency), $\omega_{j}(L)=\left[1-2\left(\alpha_{j} \cos \omega_{j}-\beta_{j} \sin \omega_{j}\right) L+\left(\alpha_{j}^{2}+\beta_{j}^{2}\right) L^{2}\right]$ (unit roots at Harmonic seasonal frequencies) for $j=1, \ldots, S^{*}$ and $\omega_{\lfloor S / 2\rfloor}(L):=\left(1+\alpha_{\lfloor S / 2\rfloor} L\right)$. The unit root hypothesis are based on these parameters. Furthermore, lag polynomial $\Psi(L)=1+\sum_{j=1}^{\infty} \phi_{j} L^{j}$ generates serial correlation in the innovations. The lag operator $L$ is defined as $L^{S j+k} y_{S n+s}=y_{S(n-j)+s-k}$. The sample size is given by $T=S N$. such that $N$ is the total number of cycles completed. Furthermore, following Eroğlu et al. (2018), we assume that initial conditions $x_{1}, \ldots, x_{S}$ are $o_{p}\left(T^{1 / 2}\right)$. However, as emphasized in del Barrio Castro et al. (2018), the limiting distributions of the test statistics will not change even if we assume stationary, possibly correlated initial conditions.

In this framework, the vector of seasons innovation process $\left\{\varepsilon_{n}\right\}$ is the source of seasonal non-stationarity behavior of the model. We define it as $\varepsilon_{n}=\left[\varepsilon_{S n-(S-1)}, \ldots, \varepsilon_{S n}\right]^{\top}$. Throughout the paper, the following set of assumptions will be taken to hold:

\section{Assumption}

$\mathcal{A} .1$ Let $\varepsilon_{n}=\Omega_{n} E_{n}$, such that $E_{n}=\operatorname{diag}\left\{e_{S n-(S-1)}, \ldots, e_{S n}\right\}$ and $\Omega_{n}=$ $\operatorname{diag}\left\{\sigma_{S n-(S-1)}, \ldots, \sigma_{S n}\right\}$ is an $S \times S$ non-stochastic matrix.

A.2 The volatility term $\Omega_{n}$ satisfies $\Omega_{n}=\Omega(n / N):=\operatorname{diag}\left\{\sigma_{(1-S)}(n / N), \ldots\right.$, $\left.\sigma_{0}(n / N)\right\}$ for all $n \in\{1, \ldots, N\}$, where $\Omega(.) \in D_{R^{S \times S}}[0,1]$, where $D_{R^{S \times S}}[0,1]$ denotes the space of $m \times n$ real matrices of cádlág function on $[0,1]$.

$\mathcal{A} .3$ Time varying unconditional variance matrix $\Upsilon(u)=\Omega(u) \Omega(u)^{\top}$ is assumed to be positive definite for all $u \in[0,1]$.

$\mathcal{A} .4$ The power series $\Psi(z)$ satisfies $\Psi(z) \neq 0$ for all $|z| \leq 1$, and $\sum_{j=0}^{\infty} j^{h}\left|\psi_{j}\right|<\infty$. 
Remark 1 Assumptions $\mathcal{A} .1-\mathcal{A} .3$ are the conditions for the innovations to display periodic non-stationary unconditional volatility. They imply that the time-varying pattern in the volatility can be observed in either some or the entire seasons. Moreover, the time-varying variance of the seasonal vector of shocks $\varepsilon_{n}$ is bounded, deterministic and exhibits a countable amount of jumps by permitting single or multiple variance shifts. Also, alteration of the PH behavior of the shocks is allowed through the sample. It is important to note that the pattern of PH where $\Omega_{n}=\operatorname{diag}\left\{\sigma_{(1-S)}, \ldots, \sigma_{0}\right\}$ is the special case of the volatility setup defined in Assumption $\mathcal{A}$. For the standard homoscedastic case, we define $\Omega_{n}=\sigma I_{S}$.

Remark 2 Assumption $\mathcal{A} .4$ implies stationarity of the innovation term $u_{S n+s}$. In other words, the only source of non-stationary dynamics is the seasonal AR polynomial $\alpha(L)$.

\section{Asymptotic analysis of FSVR tests under periodic non-stationary volatility}

In this section, we investigate the asymptotic properties of the non-parametric FSVR tests of Eroğlu et al. (2018) under the assumption of heteroscedasticity. In this regard, we first review the family of the FSVR tests, which are defined for different frequencies. Accordingly, this section mostly follows Eroğlu et al. (2018). In this family of tests, we need three important types of transformation. The first one is required to convert the time series $x_{S n+s}$ into a sub-seasonal vector process such that $X_{n}=\left[x_{S n-(S-1)}, x_{S n-(S-2)}, \ldots, x_{S n}\right] \top:=\left[X_{1, n}, X_{2, n}, \ldots, X_{S, n}\right]$ where $\forall i \in\{1, \ldots, S\} X_{i, n}$ is the $i^{t h}$ element of $X_{n}$. Next, we apply the fractional integration operator on each element of $X_{n}$ with fractional integration parameter $d>0$, such that, $\forall i \in\{1, \ldots, S\}$

$$
\tilde{X}_{i, n}=\Delta_{+}^{-d} X_{i, n}=\sum_{l=0}^{n-1} \frac{\Gamma(l+d)}{\Gamma(d) \Gamma(l+1)} X_{i, n-l},
$$

where we can define $\tilde{X}_{n}=\left[\tilde{X}_{1, n}, \ldots, \tilde{X}_{S, n}\right]^{\top}:=\left[\tilde{x}_{S n-(S-1)}, \tilde{x}_{S n-(S-2)}, \ldots, \tilde{x}_{S n}\right] \top$. The last definition can be considered as the reverse transformation of the vector process $\tilde{X}_{n}$ into a univariate process $\tilde{x}_{S n-s}$ for $n \in\{1, \ldots, n\}$ and $s \in\{(S-1), \ldots, 0\}$. Finally, we employ the HEGY transformations on the observed series $x_{S n+s}$ and $\tilde{x}_{S n+s}$, as it follows,

$$
\begin{aligned}
& x_{0, S n+s}:= \sum_{j=0}^{S-1} x_{S n+s-j}, \quad \tilde{x}_{0, S n+s}:=\sum_{j=0}^{S-1} \tilde{x}_{S n+s-j}, \\
& x_{S / 2, S n+s}:=\sum_{j=0}^{S-1} \cos [(j+1) \pi] x_{S n+s-j}, \quad \tilde{x}_{S / 2, S n+s}:=\sum_{j=0}^{S-1} \cos [(j+1) \pi] \tilde{x}_{S n+s-j},
\end{aligned}
$$




$$
\begin{aligned}
x_{i, S n+s}:= & \sum_{j=0}^{S-1} \cos \left[(j+1) \omega_{i}\right] x_{S n+s-j}, \\
\tilde{x}_{i, S n+s} & :=\sum_{j=0}^{S-1} \cos \left[(j+1) \omega_{i}\right] \tilde{x}_{S n+s-j} \quad \text { for all } i \in\left\{1, \ldots, S^{*}\right\}, \\
x_{i, S n+s}^{*}:= & \sum_{j=0}^{S-1} \sin \left[(j+1) \omega_{i}\right] x_{S n+s-j} \\
, \tilde{x}_{i, S n+s}^{*} & :=\sum_{j=0}^{S-1} \sin \left[(j+1) \omega_{i}\right] \tilde{x}_{S n+s-j} \quad \text { for all } i \in\left\{1, \ldots, S^{*}\right\} .
\end{aligned}
$$

Before constructing the test statistics, we present the relevant hypothesis. As mentioned previously, the unit root hypothesis are based on the roots of the factorized seasonal lag function. Accordingly, the following equations represents the unit root hypothesis for individual frequencies.

$$
\begin{aligned}
& H_{0, i}: \alpha_{i}=1, \quad i \in\{0,\lfloor S / 2\rfloor\} \\
& H_{0, j}: \alpha_{j}=1 \quad \text { and } \beta_{j}=0, \quad j \in\left\{1, \ldots, S^{*}\right\} .
\end{aligned}
$$

For these frequencies, we consider the test statistics of unit root on the zero, Nyquist and the harmonic frequencies by using the HEGY transformed series, as following,

$$
\begin{aligned}
& \tau_{k}(d)=N^{2 d} \frac{\sum_{n=1}^{N} \sum_{s=1-S}^{0} x_{k, S n+s}^{2}}{\sum_{n=1}^{N} \sum_{s=1-S}^{0} \tilde{x}_{k, S n+s}^{2}} \text { for } k \in\{0,\lfloor S / 2\rfloor\} . \\
& \tau_{j}(d)=N^{2 d} \frac{\sum_{t=1}^{N} \sum_{s=1-S}^{0} x_{j, S n+s}^{2}}{\sum_{n=1}^{N} \sum_{s=1-S}^{0} \tilde{x}_{j, S n+s}^{2}} \text { for } j \in\left\{1, . ., S^{*}\right\} \text {, } \\
& \tau_{j}^{*}(d)=N^{2 d} \frac{\sum_{n=1}^{N} \sum_{s=1-S}^{0} x_{j, S n+s}^{* 2}}{\sum_{n=1}^{N} \sum_{s=1-S}^{0} \tilde{x}_{j, S n+s}^{* 2}} \text { for } j \in\left\{1, \ldots, S^{*}\right\},
\end{aligned}
$$

where $\tau_{j}(d)$ and $\tau_{j}^{*}(d)$ are the test statistics for the unit root hypothesis at the harmonic frequencies $\omega_{j}$ and $2 \pi-\omega_{j}$ for all $j \in\left\{1, \ldots, S^{*}\right\}$. The following test statistic is defined for jointly testing the presence of the pairs of complex unit roots:

$$
\tilde{\tau}_{j}(d)=\left(\tau_{j}(d)+\tau_{j}^{*}(d)\right) / 2 \text { for } j \in\left\{1, \ldots, S^{*}\right\} .
$$

Finally, the F type statistics for the joint test for the unit roots in the different frequencies are given as:

$$
\tau_{1, \ldots,\lfloor S / 2\rfloor}(d)=\frac{1}{S-1}\left(\tau_{\lfloor S / 2\rfloor}(d)+\sum_{j=1}^{S^{*}} \tau_{j}(d)+\sum_{j=1}^{S^{*}} \tau_{j}^{*}(d)\right)
$$




$$
\tau_{0, \ldots,\lfloor S / 2\rfloor}(d)=\frac{1}{S}\left(\tau_{0}(d)+\tau_{\lfloor S / 2\rfloor}(d)+\sum_{j=1}^{S^{*}} \tau_{j}(d)+\sum_{j=1}^{S^{*}} \tau_{j}^{*}(d) .\right)
$$

Remark 3 The test statistic in Eq. (8) corresponds to the joint unit root tests at all frequencies except the zero frequency. Further, the test statistic in Eq. (9) corresponds to the joint unit root tests at all frequencies. The following equation gives the mathematical forms of these hypothesis, respectively.

$$
\begin{aligned}
& H_{0,1 \cdots\lfloor S / 2\rfloor}: \alpha_{1}=\cdots=\alpha_{\lfloor S / 2\rfloor}=1 \text { and } \beta_{1}=\cdots=\beta_{S^{*}}=0, \\
& H_{0,0 \cdots\lfloor S / 2\rfloor}: \alpha_{0}=\cdots=\alpha_{\lfloor S / 2\rfloor}=1 \text { and } \beta_{1}=\cdots=\beta_{S^{*}}=0 .
\end{aligned}
$$

Additionally, we can characterize alternative hypothesis for the individual frequencies as follows,

$$
H_{1, i}: \alpha_{i}<1, \quad i \in\{0,\lfloor S / 2\rfloor\} ; \quad H_{1, j}: \alpha_{j}^{2}+\beta_{j}^{2}<1, \quad \forall j \in\left\{1, \ldots, S^{*}\right\} .
$$

Seasonal unit root tests of Eroğlu et al. (2018) are constructed with the assumption of homoscedasticity. Therefore these tests do not take the periodic heteroscedasticity structure of Burridge and Taylor (2001) and non-stationary volatility structure of Cavaliere and Taylor (2008) into the account. This section aims to discuss the effect of the volatility structures on the asymptotic null distributions of FSVR tests mentioned above.

First, to obtain asymptotic null distributions of the FSVR tests outlined above when the volatility process satisfies Assumption $\mathcal{A} .1-\mathcal{A}$.3, we rewrite the $\left\{x_{S n+s}\right\}$ in a vector of seasons form as follows:

$$
X_{n}=X_{n-1}+U_{n}, t \in\{1, \ldots, N\} .
$$

where $X_{n}=\left[x_{S n-(S-1)}, \ldots, x_{S n}\right]^{\top}$ and $U_{t}=\left[u_{S n-(S-1)}, \ldots, u_{S n}\right]^{\top}, n \in\{1, \ldots, N\}$. Let us define the vector error process $U_{n}$ in the vector $M A(\infty)$ representation as

$$
U_{n}=\sum_{j=0}^{\infty} \Psi_{j} \varepsilon_{t-j}
$$

where $\Psi_{j}$ are $S \times S$ matrices in the form

$$
\Psi_{0}=\left[\begin{array}{cccccc}
1 & 0 & 0 & 0 & \ldots & 0 \\
\psi_{1} & 1 & 0 & 0 & \ldots & 0 \\
\psi_{2} & \psi_{1} & 1 & 0 & \ldots & 0 \\
\psi_{3} & \psi_{2} & \psi_{1} & 1 & \ldots & 0 \\
\cdot & . & . & . & \ldots & . \\
. & . & . & . & \ldots & . \\
\cdot & . & . & . & \ldots & . \\
\psi_{S-1} & \psi_{S-2} & \psi_{S-3} & \psi_{S-4} & \ldots & 1
\end{array}\right]
$$


and for $j \in\{1,2, \ldots\}$

$$
\Psi_{j}=\left[\begin{array}{cccccc}
\psi_{j S} & \psi_{j S-1} & \psi_{j S-2} & \psi_{j S-3} & \ldots \psi_{j S-(S-1)} \\
\psi_{j S+1} & \psi_{j S} & \psi_{j S-1} & \psi_{j S-2} & \ldots \psi_{j S-(S-2)} \\
\psi_{j S+2} & \psi_{j S+1} & \psi_{j S} & \psi_{j S-1} & \ldots \psi_{j S-(S-3)} \\
\psi_{j S+3} & \psi_{j S+2} & \psi_{j S+1} & \psi_{j S} & \ldots & \psi_{j S-(S-4)} \\
\cdot & \cdot & \cdot & \cdot & \ldots & \cdot \\
\cdot & \cdot & \cdot & \cdot & \ldots & \cdot \\
\cdot & \cdot & \cdot & \cdot & \ldots & \cdot \\
\psi_{j S+(S-1)} & \psi_{j S+(S-2)} & \psi_{j S+(S-3)} & \psi_{j S+(S-4)} & \ldots & \psi_{j S}
\end{array}\right]
$$

where $\psi_{j}, j \in\{1,2, \ldots\}$, are the MA coefficients from the inverse of $\psi(z)$.

Now, we consider the following lemma, which provides our setup's first fundamental convergence result. This lemma can be found in Cavaliere et al. (2017).

Lemma 1 Let $X_{n}$ be generated by (12)-(13) and Assumptions $\mathcal{A}$ is satisfied. Then in the space of $D_{R^{m \times n}}[0,1]$,

$$
N^{-1 / 2} X_{\lfloor. N\rfloor} \Rightarrow \Psi(1) M(.),
$$

where $\Psi(1)=\sum_{j=0}^{\infty} \Psi_{j}$ and $M()=.\int_{0} \Omega(u) d W(u)$ is an $S$-dimensional continuous martingale, with $W()=.\left(W_{1-S}(.), \ldots, W_{0}(.)\right)^{\top}$, an $S$-variate standard Brownian motion process. $M($.$) is a continuous martingale vector with the integrated covariation$ equal to $\Sigma()=.\int_{0} \Omega(u) \Omega(u)^{\top} d u$.

The proof of this lemma can be found in Cavaliere et al. (2017), thus we skip its proof. For the asymptotic analysis of the fractional scaled vector of seasons data, following Eroğlu et al. (2018), we define the vector form $\tilde{X}_{n}$ as follows:

$$
\tilde{X}_{n}=\left[\Delta_{+}^{-d} x_{S n-(S-1)}, \Delta_{+}^{-d} x_{S n-(S-2)}, \ldots, \Delta_{+}^{-d} x_{S t}\right]^{\top} .
$$

The following lemma presents the fundamental convergence result of the fractional scaled vector of seasons data under the case where the error terms have non-stationary volatility:

Lemma 2 Let $X_{n}$ be generated by (12)-(13) and Assumptions $\mathcal{A} .1$ and $\mathcal{A} .2$ are satisfied. Then in the space of $D_{R^{m \times n}}[0,1]$,

$$
N^{-d-1 / 2} \tilde{X}_{\lfloor. N\rfloor} \Rightarrow \Psi(1) M_{d+1}(.),
$$

where $\Psi(1)=\sum_{j=0}^{\infty} \Psi_{j}$ and $M_{d+1}(r)=\frac{1}{\Gamma(d+1)} \int_{0}^{r}(r-t)^{d} d M(t)$ is an $S$ dimensio fractional continuous martingale, where $M($.$) is an S$ dimensional continuous martingale defined in Lemma 1.

Remark $4 M_{d+1}$ (.) can be represented as a vector variance-transformed type II fractional Brownian motion on $[0,1]$ with independent entries. Therefore, $M_{d+1}$ (.) can be represented as $M_{d+1}()=.\left[\bar{\sigma}_{1-S} W_{\eta_{1-S}, 1-S, d+1}(.), \ldots, \bar{\sigma}_{0} W_{\eta_{0}, 0, d+1}(.)\right]^{\top}$, where 
$W_{\eta_{s}, s, d+1}()=.W_{s, d+1}\left(\eta_{s}().\right)$, with the process $\eta_{s}()=.\bar{\sigma}_{s}^{-2} \int_{0} \sigma_{s}(r)^{2} d r, \bar{\sigma}_{s}:=$ $\left(\int_{0}^{1} \sigma_{s}(r)^{2} d r\right)^{1 / 2}, s=1-S, \ldots, 0 .\left\{W_{s, d+1}(.)\right\}_{s=1-S}^{0}$ are the independent type II fractional Brownian mortions. The limiting processes $\left\{W_{s, d+1}\left(\eta_{s}(.)\right\}_{s=1-S}^{0}\right.$ are a set of $S$ independent scalar variance-transformed type II fractional Brownian motions under a transformation of the time domain with the process $\eta_{s}($.$) . For discussion of$ the properties of variance-transformed type II fractional Brownian motion, see Eroğlu and Yiğit (2016).

Using the fundamental convergence results of Lemma 1 and 2, we now present the asymptotic null distributions of the FSVR test statistics under periodic non-stationary volatility:

Theorem 1 Let the assumptions of Lemma 1 hold. The statistics $\tau_{0}(d), \tau_{S / 2}(d)$, $\tau_{j}(d), \tau_{j}^{*}(d), \tilde{\tau}_{j}(d), j=1, \ldots, S^{*}, \tau_{1, \ldots,\lfloor S / 2\rfloor}(d)$ and $\tau_{0, . ., L S / 2\rfloor}(d)$ then have the following asymptotic null distributions:

$$
\begin{aligned}
\tau_{i}(d) \Rightarrow & \frac{\int_{0}^{1} B \eta, i(r)^{2} d r}{\int_{0}^{1} B_{d+1, \eta, i}(r)^{2} d r}=: \xi_{\eta, i}(d) \quad \forall i \in\{0,\lfloor S / 2\rfloor\}, \\
\tau_{j}(d) \Rightarrow & \frac{\left\{c_{j}^{\top} \Sigma(1) c_{j}\right\} \int_{0}^{1} B_{\eta, j}(r)^{2} d r+\left\{\tilde{c}_{j}^{\top} \Sigma(1) \tilde{c}_{j}\right\} \int_{0}^{1} B_{\eta, j}^{*}(r)^{2} d r}{\left\{c_{j}^{\top} \Sigma(1) c_{j}\right\} \int_{0}^{1} B_{d+1, \eta, j}(r)^{2} d r+\left\{\tilde{c}_{j}^{\top} \Sigma(1) \tilde{c}_{j}\right\} \int_{0}^{1} B_{d+1 \eta, j}^{*}(r)^{2} d r} \\
& =: \xi_{\eta, j}(d) \quad \forall j \in\left\{1, \ldots, S^{*}\right\}, \\
\tau_{j}^{*}(d) \Rightarrow & \xi_{\eta, j}(d) \quad \forall j \in\left\{1, \ldots, S^{*}\right\}, \\
\tilde{\tau}_{j}(d) \Rightarrow & \xi_{\eta, j}(d) \quad \forall j \in\left\{1, \ldots, S^{*}\right\}, \\
\tau_{1, \ldots,\lfloor S / 2\rfloor}(d) \Rightarrow & \frac{1}{S-1}\left(\xi_{\eta, S / 2}(d)+2 \sum_{j=1}^{S^{*}} \xi_{\eta, j}(d)\right)=: \xi_{\eta, 1, \ldots,\lfloor S / 2\rfloor}(d), \\
\tau_{0, \ldots,\lfloor S / 2\rfloor}(d) \Rightarrow & \frac{1}{S}\left(\xi_{\eta, 0}(d)+\xi_{\eta, S / 2}(d)+2 \sum_{j=1}^{S^{*}} \xi_{\eta, j}(d)\right)=: \xi_{\eta, 0, \ldots,\lfloor S / 2\rfloor}(d),
\end{aligned}
$$

where

$$
\begin{aligned}
B_{\eta, i}(.):= & \left(c_{i}^{\top} \Sigma(1) c_{i}\right)^{1 / 2} c_{i}^{\top} M(.), \quad \forall i \in\{0, S / 2\} \\
B_{\eta, j}(.):= & \left(c_{j}^{\top} \Sigma(1) c_{j}\right)^{1 / 2} c_{j}^{\top} M(.), \quad B_{\eta, j}^{*}(.):=\left(\tilde{c}_{j}^{\top} \Sigma(1) \tilde{c}_{j}\right)^{1 / 2} c_{j} \tilde{c}_{j}^{\top} M(.), \\
& \forall j \in\left\{1, \ldots, S^{*}\right\}
\end{aligned}
$$

and

$$
\begin{aligned}
B_{d+1, \eta, i}(.):= & \left(c_{i}^{\top} \Sigma(1) c_{i}\right)^{1 / 2} c_{i}^{\top} M_{d+1}(.), \quad \forall i \in\{0, S / 2\}, \\
B_{d+1, \eta, j}(.):= & \left(c_{j}^{\top} \Sigma(1) c_{j}\right)^{1 / 2} c_{j}^{\top} M_{d}+1(.), \quad B_{d+1, \eta, j}^{*}(.):=\left(\tilde{c}_{j}^{\top} \Sigma(1) \tilde{c}_{j}\right)^{1 / 2} \\
& \tilde{c}_{j}^{\top} M(.), \quad \forall j \in\left\{1, \ldots, S^{*}\right\}
\end{aligned}
$$


are a set of mutually independent (normalized) variance transformed standard Brownian motion processes and variance transformed type II fractional Brownian motion processes obtained by the (mutually orthogonal) selection vectors $c_{0}=$ $[1,1,1, \ldots, 1]^{\top}, c_{S / 2}=[1,-1,1,-1, . ., 1]^{\top}, c_{j}=\left[\cos \left(\omega_{k}[1-S]\right), \cos \left(\omega_{k}[2-\right.\right.$ $S]), \ldots, \cos (0)], \tilde{c}_{j}=\left[\sin \left(\omega_{k}[1-S]\right), \sin \left(\omega_{k}[2-S]\right), \ldots, \sin (0)\right], \forall j \in\left\{1, \ldots, S^{*}\right\}$.

Remark 5 For given variance profiles, the representations for the limiting null distributions of the $\tau_{0}(d)$ and $\tau_{S / 2}(d)$ given in Theorem 1 have the same functional form as limiting null distributions of nonseasonal variance ratio statistic of Eroğlu and Yiğit (2016).The limiting null distributions of the $\tau_{0}(d), \tau_{S / 2}(d), \tilde{\tau}_{j}(d), \forall j \in\left\{1, \ldots, S^{*}\right\}$, $\tau_{1 \ldots\lfloor S / 2\rfloor}(d)$ and $\tau_{0 \ldots\lfloor S / 2\rfloor}(d)$ are all free from any weak dependence nuisance parameters.

Remark 6 In contrast to limiting null distributions of $\tau_{0}(d)$ and $\tau_{S / 2}(d)$, it is seen that the limiting null distributions of the harmonic frequency statistics $\tau_{j}(d), \tau_{j}^{*}(d)$ and $\tilde{\tau}_{j}(d)$ and joint statistics $\tau_{1 \ldots\lfloor S / 2\rfloor}(d)$ and $\tau_{0 \ldots\lfloor S / 2\rfloor}(d)$ depend on the ratio of $\left\{c_{j}^{\top} \Sigma(1) c_{j}\right\}$ to $\left\{\tilde{c}_{j}^{\top} \Sigma(1) \tilde{c}_{j}\right\}$. Since this ratio is unknown and very difficult to estimate, simulation-based tests are not easy to implement. See Burridge and Taylor (2001) for detailed discussion.

Remark 7 Thus far we have considered the case where $\left\{x_{S n+s}\right\}$ admits no deterministic component. Apparently, we have trend in our specification of the model:

$$
x_{S n+s}=\mu_{S t+s}+y_{S n+s}, \quad \forall s \in\{1-S, \ldots, 0\}, \quad \text { and } t \in\{1, \ldots, N\},
$$

where $\mu_{S n+s}=\gamma^{\top} Z_{S n+s}$ and $Z_{S n+s}$ is purely deterministic and a linear combination of the spectral indicator variables corresponding to the zero and seasonal frequencies. To conduct FSVR testing under non-stationary volatility, which will be exact invariant to the elements of $\gamma$, we can focus on the most straightforward procedure through ordinary least squares (OLS) detrending or local generalized least squares (GLS) detrending as in Eroğlu et al. (2018). If the deterministic component is allowed, the resulting de-trended series is defined as:

$$
\hat{x}_{S n+s}=x_{S n+s}-\hat{\gamma}^{\top} Z_{S n+s},
$$

and FSVR tests can be constructed with the help of $\hat{x}_{S n+s}$ rather than $x_{S n+s}$. Therefore, variance transformed standard Brownian and type II fractional Brownian motions on frequencies which are defined before, are re-defined as appropriate to the deterministic scenario of interest (see Eroğlu et al. 2018 for detrending procedures). To illustrate, let us give an example for zero frequency demeaned analogue. $B_{\eta, 0, d+1}$ will be replaced by the process $B_{\eta, 0, d+1}-\int_{0}^{1} B_{\eta, 0, d+1}(u) d u$.

In the next section, we propose wild bootstrap implementations of non-parametric seasonal unit root tests of Eroğlu et al. (2018) which are robust to periodic heteroscedasticity and permanent shifts of variance of innovations. 


\section{Wild bootstrap FSVR tests}

As we demonstrate in Theorem 1, seasonal non-stationary volatility adds a time deformation, through the seasonal variance profile, to the asymptotic null distributions of the FSVR unit root test statistics. Additionally, it is seen that the limiting null distributions of the harmonic frequency statistics $\tau_{j}(d), \tau_{j}^{*}(d)$ and $\tilde{\tau}_{j}(d)$ and joint statistics $\tau_{1 \ldots\lfloor S / 2\rfloor}(d)$ and $\tau_{0 \ldots\lfloor S / 2\rfloor}(d)$ depend on the ratio of $\left\{c_{j}^{\top} \Sigma(1) c_{j}\right\}$ to $\left\{\tilde{c}_{j}^{\top} \Sigma(1) \tilde{c}_{j}\right\}$. Constructing pivotal statistics and performing a simulation-based test is not easy because this ratio is unknown and challenging to estimate. Bootstrapping is very useful in the time series literature when the simulation-based tests are not suitable.

In this section, we first propose the seasonal non-parametric wild bootstrap implementations of the FSVR tests. Basawa et al. (1991) recommend performing bootstrapping under the null hypothesis. We follow this recommendation since if it is neglected, the finite sample performance of the unit root tests deteriorates. Therefore, also by following Cavaliere and Taylor (2008), we use seasonal first difference residual wild bootstrap. Next, we show that we obtain correct p-values in the presence of periodic heteroscedasticity and non-stationary volatility with this bootstrap.

\subsection{The seasonal wild bootstrap algorithm}

The proposed seasonal wild bootstrap algorithm is formalized in what follows:

1. Obtain the FSVR test statistics, $\tau_{0}(d), \tau_{S / 2}(d), \tau_{j}(d), j=1, \ldots, S^{*}(d), \tau_{1 \ldots\lfloor S / 2\rfloor}(d)$ and $\tau_{0 . .\lfloor S / 2\rfloor}(d)$ along with $\hat{x}_{S n+s}$, where either OLS or GLS detrending is utilized to cope with the deterministic component $\mu_{S t+s}$. Define the first difference residal vector $\Delta \hat{X}_{n}=\hat{U}_{n}=\left[\hat{u}_{S n-S-1}, \hat{u}_{S n-S-2}, \ldots, \hat{u}_{S n}\right]^{\top}, \forall n \in\{1, \ldots, N\}$.

2. Generate $U_{n}^{*}=\left[u_{S n-S-1}^{*}, u_{S n-S-2}^{*}, \ldots, u_{S n}^{*}\right]^{\top}$ by utilizing the device of randomization, $u_{S n+s}^{*}=\hat{u}_{S n+s} w_{S n+s}$, where $\left\{w_{S n+s}\right\}_{n=1}^{N}$ is an i.i.d sequence with $E\left(w_{S n+s}\right)=0, E\left(w_{S n+s}^{2}\right)=1$ and $E\left(w_{S n+s}\right)<\infty$.

3. Construct the wild bootstrap sample data by performing the following recursion

$$
\Delta X_{n}^{*}=U_{n}^{*}, \quad \forall n \in\{1, \ldots, N\}
$$

where $X_{n}^{*}=\left[u_{S n-S-1}, u_{S n-S-2}, \ldots, u_{S n}\right]^{\top}$.

4. By using the sample of bootstrap, obtain the bootstrap FSVR test statistics, $\tau_{0}^{*}, \tau_{S / 2}^{*}(d), \tau_{j}^{*}(d), j=1, \ldots, S^{*}, \tau_{1}^{*} \ldots\lfloor S / 2\rfloor(d)$ and $\tau_{0}^{*} . .\lfloor S / 2\rfloor(d)$ along with $\hat{x}_{S n+s}^{*}(d)$, where either OLS or GLS detrending is utilized to cope with the deterministic component $\mu_{S t+s}$.

5. Define p-values of the bootstrap as $P_{i, T}^{*}=G_{i, T}^{*}\left(\tau_{i}(d)\right)$, for $i \in\{0, S / 2\}, P_{j, T}^{*}=$ $G_{j, T}^{*}\left(\tau_{j}(d)\right), \forall j \in\left\{1, \ldots, S^{*}\right\}, P_{1 \ldots\lfloor S / 2\rfloor, T}^{*}=G_{1 \ldots\lfloor S / 2\rfloor, T}^{*}\left(\tau_{1 \ldots\lfloor S / 2\rfloor, T}(d)\right)$, and $P_{0 \ldots\lfloor S / 2\lfloor, T}^{*}=G_{0 \ldots\lfloor S / 2\lfloor, T}^{*}\left(\tau_{0 \ldots\lfloor S / 2\lfloor, T}(d)\right)$, where $G_{k, T}^{*}($.$) , fro k \in\{0, \ldots,\lfloor S / 2\rfloor\}$, $G_{1 \ldots\lfloor S / 2\rfloor, T}^{*}($.$) and G_{1 \ldots\lfloor S / 2\rfloor, T}^{*}($ ) are the conditional (on the sample) cumulative distribution functions of $\tau_{0}^{*}, \tau_{S / 2}^{*}, \tau_{j}^{*}$, for $j \in\left\{1, \ldots, S^{*}\right\}, \tau_{1}^{*} \ldots\lfloor S / 2\rfloor$ and $\tau_{0}^{*} \ldots\lfloor S / 2\rfloor$, respectively. 
Remark 8 As mentioned in Cavaliere and Taylor (2009), the wild bootstrap resampling strategy has the critical feature that, conditionally on the actual data, the bootstrap error terms, $U_{n}^{*}$ from step 2 are serially uncorrelated. This feature is satisfied regardless of any serial correlation observed in the first difference residuals $\hat{U}_{n}$. Therefore, the bootstrap error terms, $U_{n}^{*}$ replicate the heteroskedasticity behavior in the actual shocks. It is vital to note that, since the increments of bootstrap samples are (conditionally) serially uncorrelated, and actual FSVR tests with the corresponding asymptotic null distributions do not depend on serial correlation parameters, parametric or semiparametric correction for serial correlation is not needed for the bootstrap test statistics.

Theorem 2 Let $X_{n}$ be generated by (12)-(13) and Assumptions $\mathcal{A} .1$ and $\mathcal{A} .2$ are satisfied. Then $\tau_{i}^{*}(d) \Rightarrow \xi_{\eta, i}(d)$, for $i \in\{0, S / 2\}, \tilde{\tau}_{j}^{*}(d) \Rightarrow \xi_{\eta, j}(d)$, for $j \in\left\{1, \ldots, S^{*}\right\}$, $\tau_{1, \ldots,\lfloor S / 2\rfloor}^{*}(d) \Rightarrow \xi_{\eta, 1, \ldots,\lfloor S / 2\rfloor}(d)$ and $\tau_{1, \ldots,\lfloor S / 2\rfloor}^{*}(d) \Rightarrow \xi_{\eta, 1, \ldots,\lfloor S / 2\rfloor}(d)$, where $\Rightarrow$ denotes weak convergence in probability. Moreover, $P_{T}^{*} \stackrel{w}{\rightarrow} U[0,1]$, where $P_{T}^{*}$ denotes any of the FSVR p-values defined in seasonal wild bootstrap algorithm, and $U[0,1]$ denotes a uniform distribution on $[0,1]$.

Remark 9 Theorem 2 displays the practicality of the non-parametric seasonal wild bootstrap under the null hypothesis of the seasonal unit root tests. As the sample size goes to infinity, the bootstrapped FSVR statistics have the same asymptotic null distribution as the actual FSVR test statistics under periodic non-stationary volatility in the innovations. As a result, the bootstrap FSVR p-values have (asymptotically) uniform distribution under the null hypothesis, thus rendering the tests (asymptotically) correct-sized. Notice that the results also hold under conditional homoscedasticity since that special case is contained within Assumption 1.

\section{Finite sample simulations}

In this section, we report the finite sample size and power performance of the FSVR tests, the corresponding wild bootstrap FSVR tests, and the wild bootstrap HEGY tests. We permit the innovations to exhibit various patterns of heteroscedasticity, which are allowed under Assumption $\mathcal{A}$. The reported results are based on GLS demeaned data permitting seasonal intercepts. We place the results with OLS detrending procedure on the online appendix to save space. All experiments are based on 10,000 Monte Carlo simulations. FSVR tests are performed with $\mathrm{B}=199$ bootstrap replications. Bootstrapped variable are formed using independent standard normal variables $w_{S n+s}$. For the standard FSVR tests, critical values of Eroğlu et al. (2018) are used.

In Monte Carlo simulations, data is generated according to quarterly ( $S=4)$ DGP as follows:

$$
\begin{aligned}
\alpha(L) x_{4 n+s} & =u_{S n+s}, \\
\phi(L) u_{S n+s} & =\theta(L) \varepsilon_{S n+s}, \\
\varepsilon_{S n+s} & =\sigma_{S n+s} e_{S n+s}, \quad e_{S n+s} \text { NII } D(0,1),
\end{aligned}
$$


where $e_{S n+s}=0$, for $n \leq 0$. All simulations are conducted with $N=100$ and 0.05 significance level, with the volatility process $\sigma_{S n+s}$. We define the notation $\Sigma_{i}:=$ $\left(\sigma_{i,-3}, \sigma_{i,-2}, \sigma_{i,-1}, \sigma_{i, 0}\right), \quad \forall i \in\{0,1\}$.

We consider 3 different types of serial correlation scenarios for $u_{4 t+s}$ consistent with Eroğlu et al. (2018). First one does not contain serial correlation. For other scenarios, $u_{4 n+s}$ follows MA(4) process: $u_{4 n+s}=\left(1+0.5 L^{4}\right) \varepsilon_{4 n+s}$ and ARMA $(4,4)$ process $\left(1-0.5 L^{4}\right) u_{4 n+s}=\left(1+0.5 L^{4}\right) \varepsilon_{4 n+s}$. Moreover, we consider 6 different models of heteroscedasticity as follows:

Model 1 Constant Unconditional Volatility: $\sigma_{S n+s}^{2}=\sigma_{0}^{2}=1$.

Model 2 Periodic Heteroscedasticity: $\sigma_{S n+s}^{2}=\sigma_{0, s}^{2}$, with: Case 1, $\Sigma_{0}=(3,1,3,1)$; or Case $2, \Sigma_{0}=(30,1,1,1)$.

Model 3 Single Volatility Shift: $\sigma_{S n+s}^{2}=\sigma_{0}^{2}+\left(\sigma_{1}^{2}-\sigma_{0}^{2}\right) I(4 n+s \geq\lfloor\kappa T\rfloor)$, with $\delta:=\sigma_{0} / \sigma_{1}, \sigma_{0}=1$.

Model 4 Single Periodic Volatility Shift: $\sigma_{S n+s}^{2}=\sigma_{0, s}^{2}+\left(\sigma_{1, s}^{2}-\sigma_{0, s}^{2}\right) I(4 n+$ $s \geq\lfloor\kappa T\rfloor)$, with Case $1,\left\{\Sigma_{0}=(3,1,3,1), \Sigma_{1}=\Sigma_{0} / \delta\right\}$; Case 2, $\left\{\Sigma_{0}=(30,1,1,1), \Sigma_{1}=\Sigma_{0} / \delta\right\} ;$ Case $3,\left\{\Sigma_{0}=(1,1,1,1), \Sigma_{1}=\right.$ $(1,1 / \delta, 1,1 / \delta)\}$

Model 5 Trending Volatility: $\sigma_{S n+s}=\sigma_{0}^{2}+\left(\sigma_{1}^{2}-\sigma_{0}^{2}\right) n$,

Model 6 Exponential Integrated Stochastic Volatility $\sigma_{S n+s}^{2}=\sigma_{0}^{2} \exp (v B(r)), r \in$ $[0,1]$

In Models 3, 4 and 5 , the ratio $\delta=\sigma_{0} / \sigma_{1}$ is chosen from the values $\{1 / 3,3\}$, and the break fraction is chosen from $\kappa \in\{0.2,0.8\}$. Therefore, early and late breaks, which can be positive $(\delta<1)$ or negative $(\delta>1)$ are permitted. In the case of Model 6, following Cavaliere and Taylor (2007) we set $v=9$.

Notice that Model 4 uses a combination of the single volatility break (Model 3) of Cavaliere and Taylor (2008) and the PH pattern (Model 2) are defined in Burridge and Taylor (2001). Therefore the pattern of PH shifts at the break fraction $\kappa$. Under Cases 1 and 2 of Model 4, the magnitude of the PH alters at the break time. In Case 3 of Model 4, the shocks are homoscedastic before the break but become periodically heteroscedastic afterward. Model 1, the constant volatility case, is the benchmark to make a comparison between the finite sample performance of the actual FSVR tests and their wild bootstrap analogs when there is no heteroscedasticity.

Finally, as a robustness check we also consider the case $S=12$. In this case, we utilize above-mentioned values for the simulation setup, but consider MA(12) process $u_{12 n+s}=\left(1+0.5 L^{12}\right) \varepsilon_{12 n+s}$ and $\operatorname{ARMA}(12,12)$ process $\left(1-0.5 L^{12}\right) u_{12 n+s}=$ $\left(1+0.5 L^{12}\right)$ for the serial correlation structures.

\subsection{Size performance}

We first report the empirical size performance of the standard FSVR $\tau_{0}, \tau_{2}, \tau_{1}, \tau_{12}$, and $\tau_{12}$ tests and their wild bootstrap analogues $\tau_{0}^{*}, \tau_{2}^{*}, \tau_{1}^{*}, \tau_{12}^{*}$, and $\tau_{12}^{*}$ under the heteroskedastic shocks. The results reported in Tables 1,2,3 depict the size performances of the standard FSVR tests and their wild bootstrap analogs under GLS demeaning, with data generated according to i.i.d., ARMA(4,4), and MA(4) shocks, respectively. 
Consider first Tables 1, 2, 3 for the constant volatility case of Model 1 under different serial correlation scenarios. Here, as expected, there are no considerable distinctions between the empirical rejection frequencies (ERFs) of the standard FSVR tests and their wild bootstrap analog, particularly. Wild bootstrap is successful in replicating the homoscedastic behavior of the data.

Consider next Model 2 in these tables where there is standard PH in the innovations. This pattern observed for the standard FSVR tests is consistent with those observed in Burridge and Taylor (2001). Similar with the results of Burridge and Taylor (2001), the ERFs of the FSVR $\tau_{1}$ test (and, hence, also of the FSVR $\tau_{12}$, and $\tau_{12}$ tests) rise as the ratio of $\left(\sigma_{0,-3}^{2}+\sigma_{0,-1}^{2}\right) /\left(\sigma_{0,-2}^{2}+\sigma_{0,0}^{2}\right)$ rises, while the ERFs of the $\tau_{0}$ and $\tau_{2}$ tests are influenced regardless of the value of the aforementioned ratio. For example, in Table 1 , the size of $\tau_{1}$ under Case 1 , where PH degree is not strong, is 0.10 . However, under Case 2, where the PH degree is strong, it is 0.23 . In contrast, the bootstrap FSVR tests successfully control the size under both detrending procedures.

Consider next the results with Model 3, a single variance break. Here, significant oversizing in the standard FSVR tests is observed in the results. The most pronounced patterns of size distortions is observed for the early negative $(\delta=3$ and $\kappa=0.2)$ and late positive ( $\delta=1 / 3$ and $\kappa=0.2$ ) breaks under OLS demeaning. The oversizing problem for early negative break annihilates. However, in this case, under the late positive break, the ERFs are observed to exceed $30 \%$ for some of the tests (see Table 1). In contrast, the wild bootstrap FSVR tests show excellent control of size.

The next case we focus on is Model 4, periodic volatility shift. The results reported here are significantly consistent with those given for the standard $\mathrm{PH}$ and single variance break cases considered in Models 2 and 3, respectively. The late positive breaks create the highest size distortion on GLS demeaned tests. Under Case 3, the volatility break only influences the second and fourth quarters, and this pattern of volatility shift lowers the distortion of the size performance compared to Cases 1 and 2, where the volatility shift in the innovations influences the entire seasons. Under this model, again, the non-parametric wild bootstrap FSVR tests do a very successful job in finishing off the size distortions observed in standard FSVR tests.

Consider finally Models 5 and 6, trending volatility and exponential integrated stochastic volatility, respectively. It can be seen that for trending volatility under GLS demeaning, size distortions are observed, especially for the joint FSVR test when there is a positive $(\delta=1 / 3)$ trending volatility. The wild bootstrap FSVR tests are can handle the size control under this case. Under exponential integrated stochastic volatility for especially serially correlated error cases, there exist substantial size distortions. Again, wild bootstrap FSVR tests solve the size distortion problems under exponential integrated stochastic volatility.

We next report the empirical size performance of the non-parametric wild bootstrap FSVR tests $\tau_{0}^{*}, \tau_{2}^{*}, \tau_{1}^{*}, \tau_{12}^{*}$, and $\tau_{12}^{*}$ and the wild bootstrap HEGY tests $t_{0}^{*}, t_{2}^{*}, t_{1}^{*}, t_{12}^{*}$, and $\tau_{12}^{*}$ under the heteroskedastic innovations. The results reported in Tables 4, 5, 6 represent the size performances of the wild bootstrap FSVR and HEGY tests under GLS demeaning, with data generated according to i.i.d., ARMA(4,4), and MA(4) shocks, respectively. We observe that under GLS demeaned cases, wild bootstrap FSVR and HEGY tests display nearly similar size performance. 
We also implement the proposed testing procedure for $S=12$. Although we do not present all different scenarios for this case, the results indicate that the proposed tests perform similarly as in $S=4$. The size performance of the wild bootstrap FSVR tests is given in Table 14. This table shows that the proposed method achieves the proper size under the associated null hypothesis.

\subsection{Power performance}

We now switch to the power performance evaluation of the standard and wild bootstrap FSVR tests. Since standard and wild bootstrap FSVR tests have different size performances under periodic non-stationary volatility, we report size-adjusted powers for the standard FSVR tests for implementing a considerable comparison. Following Eroğlu et al. (2018), we take $c \in\{7,13.5\}$ under the near-seasonally integrated alternative $\alpha(L)=\left(1-(1+c / N) L^{4}\right)$ for the power evaluation.

We first investigate the power properties of the standard FSVR $\tau_{0}, \tau_{2}, \tau_{1}, \tau_{12}$, and $\tau_{12}$ tests and their non-parametric wild bootstrap analogues $\tau_{0}^{*}, \tau_{2}^{*}, \tau_{1}^{*}, \tau_{12}^{*}$, and $\tau_{12}^{*}$ under the heteroskedastic shocks. The results reported in Tables 7, 8, 9 represent the power performances of the standard FSVR tests and their wild analogs counterparts under GLS demeaning, with data generated according to i.i.d., $\operatorname{ARMA}(4,4)$, and MA(4) shocks, respectively.

The fundamental inferences obtained from the simulation results in 7,8,9 can be easily summarized as follows. In the constant volatility case (Model 1), the reported results show no significant loss in power performance with the usage of non-parametric wild bootstrap. At the same time, the wild bootstrap is very successful in solving the over-sizing problem caused by the standard FSVR tests under heteroscedasticity. Second, the power performance of both the standard and wild bootstrap FSVR tests depend on the heteroskedasticity present in the innovations. In some cases, when OLS detrending (see the tables in the online appendix) is utilized, both tests' power performance deteriorates, especially in the single volatility break case with $\delta=3$ and $\kappa=0.2$. However, under GLS demeaning, this problem disappears.

Next we report the power performance of the non-parametric wild bootstrap FSVR tests $\tau_{0}^{*}, \tau_{2}^{*}, \tau_{1}^{*}, \tau_{12}^{*}$, and $\tau_{12}^{*}$ and wild bootstrap HEGY tests $t_{0}^{*}, t_{2}^{*}, t_{1}^{*}, t_{12}^{*}$, and $t_{12}^{*}$ under the heteroskedastic innovations. The results reported in Tables 10, 11, 12 represent the power performance of the wild bootstrap FSVR and HEGY tests under GLS demeaning, with data generated according to i.i.d., $\operatorname{ARMA}(4,4)$ and MA(4) shocks, respectively. The power results are consistent with the results of Eroğlu et al. (2018). Under GLS demeaning case, wild bootstrap HEGY tests have the advantage in the individual coefficient testing relative to the wild bootstrap FSVR tests under homoscedastic and PH errors. However, under non-stationary volatility, they have similar power performance. Additionally, under GLS demeaning, the FSVR and HEGY methods enjoy similar power properties in the joint tests.

Similar to the size exercise, we investigate the power properties of the testing procedure on the case $S=12$. In Table 15, we demonstrate the size and the sizeadjusted power properties of the proposed method when $S=12$. The results are very 

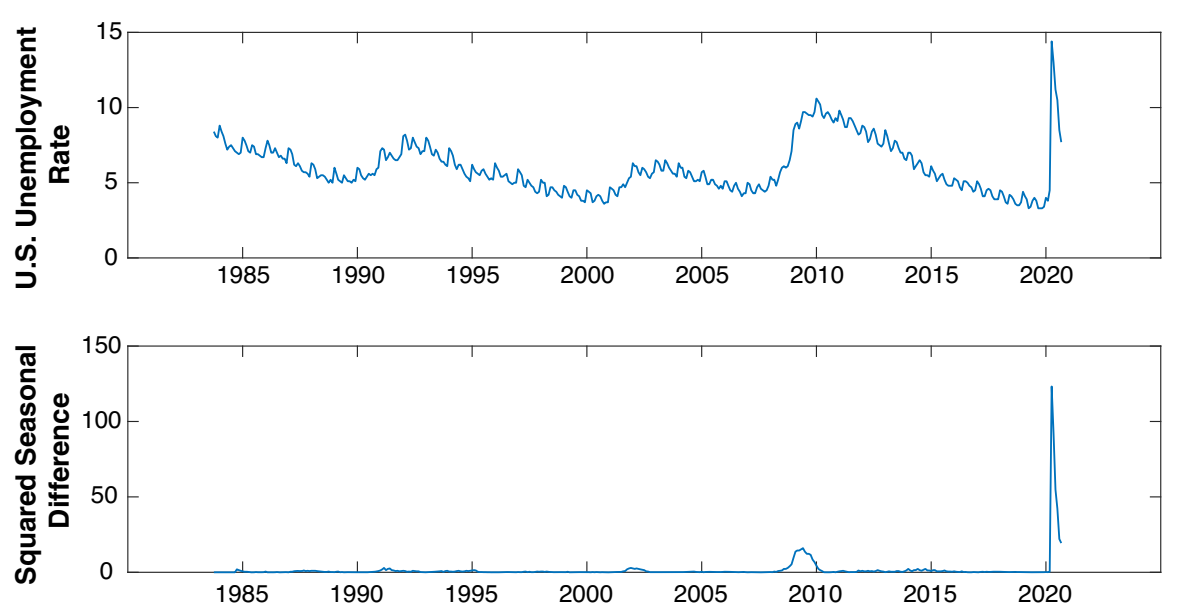

Fig. 1 The Monthly U.S Unemployment Rate between 1983 and 2020

similar to the $S=4$ scenario. Our testing procedure generates quite satisfying power properties in every design considered.

\section{Empirical results}

In this section, we apply the newly proposed seasonal unit root procedure on the monthly unemployment data of the U.S. Our dataset spans monthly data from $1983-$ $M 9$ and 2020-M9. We illustrate the Unemployment data (upper panel) and the square of its seasonal difference (lower panel) in Fig. 1. From the lower panel of the picture, we can observe volatility changes, especially during the crisis periods in 2008 and the Covid-19 pandemic period. Accordingly, using a heteroscedasticity-robust procedure can improve the inference in detecting seasonal unit roots. In addition, the data is not seasonally adjusted to capture seasonal dynamics. Since we have monthly data, we set $S=12$. In Table 13, we demonstrate p-values of two wild bootstrap test families, namely, FSVR and HEGY. In this table, we report individual tests for 0 and Nyquist $\omega_{S / 2}=\pi$ frequencies; joint tests for the harmonic $\omega_{1}=\pi / 6, \omega_{2}=\pi / 3, \omega_{3}=\pi / 2$, $\omega_{4}=2 \pi / 3$ and $\omega_{5}=5 \pi / 6$; the overall joint tests for 0 to $\pi$ and 1 to $\pi$. Moreover, we set $\mathrm{B}=9999$ to create the wild bootstrap replications and the confidence level as 0.05 .

In Table 13, FSVR and HEGY tests give slightly different results. While the FSVR test found a unit root at the zero and harmonic frequencies, HEGY tests found a unit root at the Nyquist and harmonic frequencies. These findings are also reflected in the joint overall tests. FSVR test rejects the joint presence of unit roots at all frequencies and each frequency except zero frequency. On the contrary, HEGY found the joint presence of unit roots at all frequencies except zero. 


\section{Conclusion}

In this article, we have explored the effect of a broad class of heteroscedastic models on the FSVR tests of Eroğlu et al. (2018). This class primarily covers the well-known cases of periodic heteroscedasticity and non-stationary volatility. In this regard, first, we have analyzed the impact of the periodic non-stationary volatility on the asymptotic behavior of FSVR tests. We have shown that the obtained asymptotic null distributions of the FSVR test statistics are highly affected by the nuisance parameters arising from non-stationary seasonal volatility. We have also documented the standard FSVR tests' results under different heteroscedasticity patterns. The results indicate that heteroscedastic shocks render these tests significantly oversized.

We have also demonstrated that the derived asymptotic distributions of the FSVR test statistics are nonstandard and not suitable for performing simulation-based tests. As a remedy, we have proposed a non-parametric wild bootstrap analog of the FSVR tests, which are so helpful in replicating the patterns of heteroscedasticity in the actual shocks. The bootstrap test statistics have also shown asymptotically pivotal inference. The proposed wild bootstrap FSVR tests successfully solve the size distortion problem observed in the standard FSVR tests without losing finite sample power. Lastly, we have compared the finite sample properties of wild bootstrap FSVR and parametric HEGY tests. Simulation results reveal that wild bootstrap FSVR tests have a desirable size and power properties. Finally, we employ our methodology on the monthly U.S. unemployment data. Our results indicate that wild bootstrap versions of HEGY and FSVR tests give slightly different results.

Supplementary Information The online version contains supplementary material available at https://doi. org/10.1007/s00180-022-01211-w.

\section{Appendix A: Preliminaries and proofs}

\section{Preliminaries}

In this appendix, by following Cavaliere et al. (2017), we will define some crucial definitions and make use of them. First of all, let us define $c_{0}, c_{S / 2}, c_{k}, \tilde{c}_{k}, k \in$ $\left\{1, \ldots, S^{*}\right\}$, which denote the (mutually) orthogonal $S \times 1$ selection vectors as:

$$
\begin{aligned}
c_{0} & =[1,1,1, \ldots, 1]^{\top}, \\
c_{S / 2} & =[1,-1,1,-1, . ., 1]^{\top}, \\
c_{k} & =\left[\cos \left(\omega_{k}[1-S]\right), \cos \left(\omega_{k}[2-S]\right), \ldots, \cos (0)\right]^{\top}, \\
\tilde{c}_{k} & =\left[\sin \left(\omega_{k}[1-S]\right), \sin \left(\omega_{k}[2-S]\right), \ldots, \sin (0)\right]^{\top} .
\end{aligned}
$$

We also introduce the $S \times S$ circulant matrices as follows:

$$
\begin{aligned}
& C_{0}=\operatorname{circ}[1,1,1, \ldots, 1], \quad C_{S / 2}=\operatorname{circ}[1,-1,1,-1, . ., 1], \\
& C_{i}=\operatorname{circ}\left[\cos (0), \cos \left(\omega_{i}\right), \ldots, \cos \left((S-1) \omega_{i}\right)\right] \quad \omega_{i}=2 \pi i / S, \quad \forall i \in\left\{1, \ldots, S^{*}\right\},
\end{aligned}
$$




$$
\tilde{C}_{i}=\operatorname{circ}\left[\sin (0), \sin \left(\omega_{i}\right), \ldots, \sin \left((S-1) \omega_{i}\right)\right] \quad \omega_{i}=2 \pi i / S, \quad \forall i \in\left\{1, \ldots, S^{*}\right\}
$$

As discussed in Smith et al. (2009) and Cavaliere et al. (2017), these matrices are mutually orthogonal and have the following properties: $C_{0} C_{0}=S C_{0}, C_{S / 2} C_{S / 2}=$ $S C_{S / 2}, C_{j} \tilde{C}_{j}=(S / 2) \tilde{C}_{j}, \tilde{C}_{j} \tilde{C}_{j}=(S / 2) C_{j}$, for $j \in\left\{1, \ldots, S^{*}\right\}$. Also, the relationship between vectors and circulant matrices are: $C_{0}=c_{0} c_{0}^{\top}, C_{S / 2}=c_{S / 2} c_{S / 2}^{\top}, C_{j}=c_{j} c_{j}^{\top}$, $\tilde{C}_{j}=c_{j} \tilde{c}_{j}^{\top}$, where

$$
\begin{aligned}
& c_{j}=\left(\begin{array}{c}
\cos \left(\omega_{j}[1-S]\right), \cos \left(\omega_{j}[2-S]\right), \ldots, \cos (0) \\
\sin \left(\omega_{j}[1-S]\right), \sin \left(\omega_{j}[2-S]\right), \ldots, \sin (0)
\end{array}\right)^{\top} \\
& \tilde{c}_{j}=\left(\begin{array}{cc}
-\sin \left(\omega_{j}[1-S]\right), & -\sin \left(\omega_{j}[2-S]\right), \ldots,-\sin (0) \\
\cos \left(\omega_{j}[1-S]\right), & \cos \left(\omega_{j}[2-S]\right), \ldots, \cos (0)
\end{array}\right)^{\top}
\end{aligned}
$$

Now, we will present the proof of Lemma 2:

Proof of Lemma 2 We know from Boswijk et al. (2016) for $\varepsilon_{t}=\Omega_{t} E_{t}$, the following convergence result holds:

$$
Y_{\lfloor. N\rfloor}=N^{-1 / 2} \sum_{t=1}^{\lfloor. N\rfloor} \varepsilon_{t} \Rightarrow M(.) .
$$

Now, first we need to prove the convergence result of the following fractional partial sum:

$$
N^{-1 / 2-d} \sum_{t=1}^{\lfloor\cdot N\rfloor} \Delta_{+}^{-d} \varepsilon_{t} .
$$

We know from Nielsen (2010) Lemma 6.d that $\Delta_{+}^{-d}$ satisfies $\Delta_{+}^{-d} \Delta_{+}^{-d_{2}} Y_{t}=$ $\Delta_{+}^{-d_{2}} \Delta_{+}^{-d} Y_{t}=\Delta_{+}^{-d-d_{2}} Y_{t}$, we can write

$$
\sum_{i=1}^{r} \Delta_{+}^{-d} \varepsilon_{i}=\Delta_{+}^{-d} \sum_{i=1}^{r} \varepsilon_{i}
$$

Therefore,

$$
\begin{aligned}
N^{-1 / 2-d} \sum_{t=1}^{\lfloor r N\rfloor} \Delta_{+}^{-d} \varepsilon_{t}, & =N^{-1 / 2-d} \sum_{t=1}^{\lfloor r N\rfloor} \pi_{\lfloor r N\rfloor-t}(d) \sum_{i=1}^{t} \varepsilon_{i}, \\
& =N^{-1 / 2-d} \sum_{t=1}^{\lfloor r N\rfloor} \pi_{\lfloor r N\rfloor-t}(d+1) \varepsilon_{t}, \\
& =N^{-1 / 2-d} \sum_{t=1}^{\lfloor r N\rfloor} \frac{\left(\left\lfloorr N\lfloor-t)^{d}\right.\right.}{\Gamma(d+1)} \varepsilon_{t},
\end{aligned}
$$




$$
\begin{aligned}
& =\sum_{t=1}^{\lfloor r N\rfloor} \frac{(r-t / N)^{d}}{\Gamma(d+1)} N^{-1 / 2} \varepsilon_{t}, \\
& =\sum_{t=1}^{\lfloor r N\rfloor} \int_{(r-1) / N}^{r / N} \frac{(r-t / N)^{d}}{\Gamma(d+1)} d y_{N}(s), \\
& \Rightarrow \frac{1}{\Gamma(d+1)} \int_{0}^{r}(r-t)^{d} d M(t)=M_{d+1}(r) .
\end{aligned}
$$

Consider now, $\tilde{X}_{\lfloor r N\rfloor}=\sum_{t=1}^{\lfloor r N\rfloor} \Delta_{+}^{-d} U_{t}$. By Beveridge Nelson decomposition, we have $U_{t}=\Psi(1) \varepsilon_{t}+\varepsilon_{t-1}{ }^{*}-\varepsilon_{t}{ }^{*}$, where $\varepsilon_{t}{ }^{*}=\Psi^{*}(L) \varepsilon_{t}$. Now we have

$$
N^{-1 / 2-d} \sum_{t=1}^{\lfloor r N\rfloor} \Delta_{+}^{-d} U_{t}=\Psi(1) N^{-1 / 2-d} \sum_{t=1}^{\lfloor r N\rfloor} \Delta_{+}^{-d} \varepsilon_{t}+N^{-1 / 2-d} \Delta_{+}^{-d}\left(\varepsilon_{0}^{*}-\varepsilon\lfloor r N\rfloor{ }^{*}\right) .
$$

Under Assumptions $\mathcal{A} .1$ and $\mathcal{A} .2$, the second term on the above equation is $O_{p}\left(N^{(-1-2 d)}\right)$ uniformly in $r \in[0,1]$. Therefore, we have the desired result that

$$
N^{-d-1 / 2} \tilde{X}_{\lfloor. N\rfloor} \Rightarrow \Psi(1) M_{d+1}(.)
$$

Proof of Theorem 1 Now, we will obtain the limiting distribution of FSVR tests under non-stationary volatility. Firstly, notice that using Boswijk et al. (2016), Lemma 1, and CMT, the following results hold:

$$
\begin{gathered}
N^{-1} \sum_{t=2}^{N} X_{t-1} X_{t-1}^{\top} \Rightarrow \Psi(1) \int_{0}^{1} M(r) M(r)^{\top} d r \Psi(1)^{\top}=: Q_{1}, \\
N^{-1-2 d} \sum_{t=2}^{N} \tilde{X}_{t-1} \tilde{X}_{t-1}^{\top} \Rightarrow \Psi(1) \int_{0}^{1} M_{d+1}(r) M_{d+1}(r)^{\top} d r \Psi(1)^{\top}=: Q_{2} .
\end{gathered}
$$

Now, firstly consider the numerator of $\tau_{j}(d)$, for $i \in\{0, S / 2\}$. By standard manipulations, Cavaliere et al. (2017) show that

$$
\begin{aligned}
& T^{-2} \sum_{t=2}^{N} \sum_{S=1-S}^{0} x_{j, S t+s}^{2} \Rightarrow \frac{1}{S} c_{j}^{\top} \Psi(1) \int_{0}^{1} M(r) M(r)^{\top} d r \Psi(1)^{\top} c_{j}=\frac{1}{S} c_{j}^{\top} Q_{1} c_{j}, \\
& \forall j \in\{0, S / 2\} .
\end{aligned}
$$


Similar manipulations for the harmonic pairs can be done with the denominator as it is shown in Proposition 1 of Cavaliere et al. (2017):

$$
\begin{aligned}
T^{-2} \sum_{t=2}^{N} \sum_{s=1-S}^{0}\left(x_{j, S t+s}\right)^{2} & \Rightarrow \frac{1}{2 S}\left(c_{j}^{\top} \Psi(1) \int_{0}^{1} M(r) M(r)^{\top} d r \Psi(1)^{\top} c_{j}+\tilde{c}_{j}^{\top}\right. \\
& \left.\Psi(1) \int_{0}^{1} M(r) M(r)^{\top} d r \Psi(1)^{\top} \tilde{c}_{j}\right), \\
& =\frac{1}{2 S}\left[c_{j}^{\top} Q_{1} c_{j}+\tilde{c}_{j}^{\top} Q_{1} \tilde{c}_{j}\right], \quad \forall j \in\left\{1, \ldots, S^{*}\right\},
\end{aligned}
$$

and

$$
T^{-2} \sum_{t=2}^{N} \sum_{s=1-S}^{0}\left(x_{j, S t+s}^{*}\right)^{2} \Rightarrow \frac{1}{2 S}\left[c_{j}^{\top} Q_{1} c_{j}+\tilde{c}_{j}^{\top} Q_{1} \tilde{c}_{j}\right], \quad \forall j \in\left\{1, \ldots, S^{*}\right\} .
$$

Now, we focus on the denominators. For zero and Nyquist frequency, we have:

$$
\begin{aligned}
T^{-2}-2 d \sum_{t=2}^{N} \sum_{s=1-S}^{0} \tilde{x}_{j, S t+s}^{2} \\
=T^{-2-2 d} \sum_{t=2}^{N} \tilde{X}_{j, t}^{\top} \tilde{X}_{j, t}=T^{-2-2 d} \sum_{n=2}^{N} S\left(\tilde{X}_{t-1}^{\top} C_{j} \tilde{X}_{t-1}\right)+o_{p}(1), \\
=T^{-2-2 d} \sum_{t=2}^{N} S\left(\tilde{X}_{t-1}^{\top} c_{j} c_{j}^{\top} \tilde{X}_{t-1}\right)+o_{p}(1), \\
=T^{-2-2 d} \sum_{t=2}^{N} t r\left\{S\left(c_{j}^{\top} \tilde{X}_{t-1} \tilde{X}_{t-1}^{\top} c_{j}\right\}+o_{p}(1,)\right. \\
=S c_{j}^{\top} T^{-2-2 d} \sum_{t=2}^{N} \tilde{X}_{t-1} \tilde{X}_{t-1}^{\top} c_{j}+o_{p}(1), \\
\Rightarrow \frac{1}{S} c_{j}^{\top} \Psi(1) \int_{0}^{1} M_{d+1}(r) M_{d+1}(r)^{\top} d r \Psi(1)^{\top} c_{j}, \\
=\frac{1}{S} c_{j}^{\top} Q_{2} c_{j}, \quad \forall j \in\{0, S / 2\} .
\end{aligned}
$$

For the harmonic frequency pairs, we have

$$
\begin{gathered}
T^{-2-2 d} \sum_{t=2}^{N} \sum_{S=1-S}^{0}\left(\tilde{x}_{j, S t+s}\right)^{2}=T^{-2-2 d} \sum_{t=2}^{N} \tilde{X}_{j, t} \tilde{X}_{j, t} \\
=T^{-2-2 d} \sum_{t=2}^{N}(S / 2)\left(\tilde{X}_{t-1}^{\top} C_{j} \tilde{X}_{t-1}\right)+o_{p}(1),
\end{gathered}
$$




$$
\begin{aligned}
& =T^{-2-2 d} \sum_{t=2}^{N}(S / 2)\left(\tilde{X}_{t-1}^{\top} c_{j} c_{j}^{\top} \tilde{X}_{t-1}\right)+o_{p}(1), \\
& =T^{-2-2 d} \sum_{t=2}^{N}(S / 2) \operatorname{tr}\left\{\left(c_{j}^{\top} \tilde{X}_{t-1} \tilde{X}_{t-1}^{\top} c_{j}\right)\right\}+o_{p}(1), \\
& =T^{-2-2 d} \sum_{t=2}^{N}(S / 2) \operatorname{tr}\left\{\left(\begin{array}{l}
c_{j}^{\top} \\
\tilde{c}_{j}^{\top}
\end{array}\right) \tilde{X}_{t-1} \tilde{X}_{t-1}^{\top}\left(c_{j} \tilde{c}_{j}\right)\right\}+o_{p}(1), \\
& =T^{-2-2 d} \sum_{t=2}^{N}(S / 2) \operatorname{tr}\left(\begin{array}{l}
c_{j}^{\top} \tilde{X}_{t-1} \tilde{X}_{t-1}^{\top} c_{j} \quad c_{j}^{\top} \tilde{X}_{t-1} \tilde{X}_{t-1}^{\top} \tilde{X}_{t-1}^{\top} c_{j} \tilde{c}_{j}, \\
\tilde{c}_{j}^{\top} \tilde{X}_{t-1} \tilde{X}_{t-1}^{\top} \tilde{c}_{j},
\end{array}\right)+o_{p}(1) \\
& =(S / 2) T T^{-2-2 d} \sum_{t=2}^{N}\left(c_{j}^{\top} \tilde{X}_{t-1} \tilde{X}_{t-1}^{\top} c_{j}+\tilde{c}_{j}^{\top} \tilde{X}_{t-1} \tilde{X}_{t-1}^{\top} \tilde{c}_{j}\right)+o_{p}(1), \\
& \Rightarrow \frac{1}{2 S}\left(c_{j}^{\top} \Psi(1) \int_{0}^{1} M_{d+1}(r) M_{d+1}(r)^{\top} d r \Psi(1)^{\top} c_{j},\right. \\
& \left.+\tilde{c}_{j}^{\top} \Psi(1) \int_{0}^{1} M_{d+1}(r) M_{d+1}(r)^{\top} d r \Psi(1)^{\top} \tilde{c}_{j}\right), \\
& =\frac{1}{2 S}\left[c_{j}^{\top} Q_{2} c_{j}+\tilde{c}_{j}^{\top} Q_{2} \tilde{c}_{j}\right], \quad \forall j \in\left\{1, \ldots, S^{*}\right\},
\end{aligned}
$$

and

$$
\begin{aligned}
T^{-2-2 d} \sum_{t=2}^{N} \sum_{s=1-S}^{0}\left(\tilde{x}_{j, S t+s}^{*}\right)^{2} & =T^{-2-2 d} \sum_{t=2}^{N} \tilde{X}_{j, t}^{*} \tilde{X}_{j, t}^{*} \\
& =T^{-2-2 d} \sum_{t=2}^{N}(S / 2)\left(\tilde{X}_{t-1}^{\top} \tilde{C}_{j} \tilde{C}_{j} \tilde{X}_{t-1}\right)+o_{p}(1), \\
& =T^{-2-2 d} \sum_{t=2}^{N}(S / 2)\left(\tilde{X}_{t-1}^{\top} \tilde{C}_{j} \tilde{X}_{t-1}\right)+o_{p}(1) \\
& \Rightarrow \frac{1}{2 S}\left[c_{j}^{\top} \tilde{Q} c_{j}+\tilde{c}_{j}^{\top} \tilde{Q}_{j}\right], \quad \forall j \in\left\{1, \ldots, S^{*}\right\}
\end{aligned}
$$

Now, with the help of the S(normalized) variance transformed Brownian and type II fractional variance transformed Brownian motion processes, firstly, let us consider limiting distribution of $\tau_{0}(d)$ statistic under periodic non-stationary volatility:

$$
\begin{aligned}
\tau_{0}(d) & \Rightarrow \frac{c_{0}^{\top} Q_{1} c_{0} c_{0}^{\top} \Sigma(1) c_{0}}{c_{0}^{\top} Q_{2} c_{0} c_{0}^{\top} \Sigma(1) c_{0}}=\frac{c_{0}^{\top} \Psi(1) Q_{1}^{*} \Psi(1)^{\top} c_{0} c_{0}^{\top} \Sigma(1) c_{0}}{c_{0}^{\top} \Psi(1) Q_{2}^{*} \Psi(1)^{\top} c_{0} c_{0}^{\top} \Sigma(1) c_{0}} \\
& =\frac{\psi(1) c_{0}^{\top} Q_{1}^{*} c_{0} \psi(1) c_{0}^{\top} \Sigma(1) c_{0}}{\psi(1) c_{0}^{\top} Q_{2}^{*} c_{0} \psi(1) c_{0}^{\top} \Sigma(1) c_{0}},
\end{aligned}
$$




$$
=\frac{c_{0}^{\top}\left(\int_{0}^{1} M(r) M(r)^{\top} d r\right) c_{0} c_{0}^{\top} \Sigma(1) c_{0}}{c_{0}^{\top}\left(\int_{0}^{1} M_{d+1}(r) M_{d+1}(r)^{\top} d r\right) c_{0} c_{0}^{\top} \Sigma(1) c_{0}}=\frac{\int_{0}^{1} B_{\eta, 0}^{2}(r) d r}{\int_{0}^{1} B_{d+1, \eta, 0}^{2}(r) d r} .
$$

The preceding result comes from the result of del Barrio Castro et al. (2012) such that $c_{0}^{\top} \Psi(1)=\psi(1) c_{0}$. The proof for the limiting distribution of $\tau_{S / 2}(d)$ at Nyquist frequency is similar to the zero frequency except the corresponding result of del Barrio Castro et al. (2012) such that $c_{S / 2}^{\top} \Psi(1)=\psi(-1) c_{S / 2}$ and that $c_{S / 2}^{\top} \Sigma(1) c_{S / 2}=$ $c_{0}^{\top} \Sigma(1) c_{0}$.

For the frequency pair limiting distribution, let us introduce the results of del Barrio Castro et al. (2012). We have $c_{k}^{\top} \Psi(1)=a_{k} \tilde{c}_{k}^{\top}+b_{k} c_{k}^{\top}$ and $\tilde{c}_{k}^{\top} \Psi(1)=b_{k} \tilde{c}_{k}^{\top}-a_{k} c_{k}^{\top}$ where $a_{k}=\operatorname{Im}\left(\psi\left(\exp \left(i \omega_{k}\right)\right)\right.$ and $b_{k}=\operatorname{Re}\left(\psi\left(\exp \left(i \omega_{k}\right)\right)\right.$, for $k \in\left\{1, \ldots, S^{*}\right\}, \operatorname{Re}()$. and $\operatorname{Im}($.$) denoting the real and imaginary parts of their arguments, respectively.$ Therefore, for $k \in\left\{1, . ., S^{*}\right\}$, we have

$$
\begin{aligned}
& c_{k}^{\top} Q_{1} c_{k}=\left(a_{k} \tilde{c}_{k}^{\top}+b_{k} c_{k}^{\top}\right)\left(\int_{0}^{1} M(r) M(r)^{\top} d r\right)\left(a_{k} \tilde{c}_{k}^{\top}+b_{k} c_{k}^{\top}\right), \\
& \tilde{c}_{k}^{\top} Q_{1} \tilde{c}_{k}=\left(b_{k} \tilde{c}_{k}^{\top}-a_{k} c_{k}^{\top}\right)\left(\int_{0}^{1} M(r) M(r)^{\top} d r\right)\left(b_{k} \tilde{c}_{k}^{\top}-a_{k} c_{k}^{\top}\right), \\
& c_{k}^{\top} Q_{2} c_{k}=\left(a_{k} \tilde{c}_{k}^{\top}+b_{k} c_{k}^{\top}\right)\left(\int_{0}^{1} M_{d+1}(r) M_{d+1}(r)^{\top} d r\right)\left(a_{k} \tilde{c}_{k}^{\top}+b_{k} c_{k}^{\top}\right), \\
& \tilde{c}_{k}^{\top} Q_{2} \tilde{c}_{k}=\left(b_{k} \tilde{c}_{k}^{\top}-a_{k} c_{k}^{\top}\right)\left(\int_{0}^{1} M_{d+1}(r) M_{d+1}(r)^{\top} d r\right)\left(b_{k} \tilde{c}_{k}^{\top}-a_{k} c_{k}^{\top}\right) .
\end{aligned}
$$

The final step for obtaining the limiting distribution for frequency pair is as follows:

$$
\begin{aligned}
& c_{k}^{\top} Q_{1} c_{k}+\tilde{c}_{k}^{\top} Q_{1} \tilde{c}_{k}=\frac{S}{2}\left(a_{k}^{2}+b_{k}^{2}\right)\left(\left\{c_{k}^{\top} \Sigma(1) c_{k}\right\}\right. \\
& \left.\int_{0}^{1} B_{\eta, k}(r)^{2} d r+\left\{\tilde{c}_{k}^{\top} \Sigma(1) \tilde{c}_{k}\right\} \int_{0}^{1} B_{\eta, k}(r)^{2 *} d r\right), \\
& c_{k}^{\top} Q_{2} c_{k}+\tilde{c}_{k}^{\top} Q_{2} \tilde{c}_{k}=\frac{S}{2}\left(a_{k}^{2}+b_{k}^{2}\right)\left(\left\{c_{k}^{\top} \Sigma(1) c_{k}\right\}\right. \\
& \left.\int_{0}^{1} B_{d+1, \eta, k}(r)^{2} d r+\left\{\tilde{c}_{k}^{\top} \Sigma(1) \tilde{c}_{k}\right\} \int_{0}^{1} B_{d+1, \eta, k}(r)^{2 *} d r\right) .
\end{aligned}
$$

The stated results for $\tau_{k}(d), \forall k \in\left\{1, \ldots, S^{*}\right\}$ statistics then follow after some routine algebra.

Proof of Theorem 2 Let us define $S_{N}^{b}(r)=N^{-1 / 2} X_{\lfloor r N\rfloor}^{*}$, where $U_{n}^{*}=\left[u_{S n-(S-1)}^{*}\right.$, $\left.u_{S n-(S-2)}^{*}, \ldots, u_{S n}^{*}\right]^{\top}$. We can express $U_{n}^{*}$ by definition as $U_{n}^{*}=\hat{U}_{n} \cdot w_{n}=(\Psi(L)$ $\left.\hat{E}_{n}\right) w_{n}$, where $\Psi(L)$ is the lag polynomial that creates serial correlation. By Cavaliere and Taylor (2009), we know that wild bootstrap kills the serial correlation in the innovations. Therefore, we have $U_{n}^{*}=\hat{E}_{n} w_{n}=E_{n}^{*}$. 
Under Assumption $\mathcal{A}$, by using by Lemma 4 of Boswijk et al. (2016), we have

$$
S_{N}^{b}(r)=N^{-1 / 2} \sum_{n=2}^{\lfloor r N\rfloor} U_{n}^{*} \Rightarrow M(r),
$$

where $U_{n}^{*}=\left[u_{S n-(S-1)}^{*}, u_{S n-(S-2)}^{*}, \ldots, u_{S n}^{*}\right]^{\top}$, because conditional on $\left\{\hat{U}_{n}\right\}_{n=1}^{N}$, $S_{N}^{b}(r)$ is a Gaussian process with independent increments and covariance kernel $E *\left(S_{N}^{b}(.) S_{N}^{b}().\right)=\sum_{n=2}^{\lfloor r N\rfloor} \hat{E}_{n} \hat{E}_{n}^{\top}$, where $\sum_{n=2}^{\lfloor r N\rfloor} \hat{E}_{n} \hat{E}_{n}^{\top} \rightarrow \Sigma(r)$ in probability uniformly for all $r \in[0,1]$.

Now consider $\tilde{S}_{N}^{b}(r)=N^{-1 / 2-d} \tilde{X}_{\lfloor r N\rfloor}^{*}$, where $\tilde{U}_{n}^{*}=\left[\tilde{u}_{S n-(S-1)}^{*}, \tilde{u}_{S n-(S-2)}^{*}, \ldots\right.$, $\left.\tilde{u}_{S n}^{*}\right]^{\top}$. Now, we can write $\tilde{U}_{n}^{*}$ as $\tilde{U}_{n}^{*}=\Delta_{+}^{-d} U_{n}^{*}=\Delta_{+}^{-d}\left(\hat{U}_{n} \cdot w_{n}\right)=\Delta_{+}^{-d}\left(\hat{E}_{n} \cdot w_{n}\right)=$ $\Delta_{+}^{-d} E_{n}^{*}$

Now, Under Assumption $\mathcal{A}$, by using the main result in Lemma 1, and (21), we can conclude that

$$
\tilde{S}_{N}^{b}(r)=N^{-1 / 2-d} \sum_{n=2}^{\lfloor r N\rfloor} \tilde{U}_{n}^{*} \Rightarrow M_{d+1}(r) .
$$

Now, by using the main convergence results (21) and (22) related to the wild bootstrap, we can continue as follows:

$$
\begin{gathered}
N^{-1} \sum_{t=2}^{N} X_{t-1}^{*} X_{t-1}^{*} \Rightarrow \int_{0}^{1} M(r) M(r)^{\top} d r=: Q_{1}^{*}, \\
N^{-1-2 d} \sum_{t=2}^{N} \tilde{X}_{t-1}^{*} \tilde{X}_{t-1}^{*} \Rightarrow \int_{0}^{1} M_{d+1}(r) M_{d+1}(r)^{\top} d r=: Q_{2}^{*},
\end{gathered}
$$

Therefore, we obtain

$$
\begin{aligned}
& T^{-2} \sum_{t=2}^{N} \sum_{s=1-S}^{0} x_{j, S t+s^{*}}^{2} \Rightarrow \frac{1}{S} c_{j}^{\top} Q_{1}^{*} c_{j}, \quad \forall j \in\{0, S / 2\}, \\
& T^{-2} \sum_{t=2}^{N} \sum_{s=1-S}^{0}\left(x_{j, S t+s}^{* *}\right)^{2} \Rightarrow \frac{1}{2 S}\left[c_{j}^{\top} Q_{1}^{*} c_{j}+\tilde{c}_{j}^{\top} Q_{1}^{*} \tilde{c}_{j}\right], \quad \forall j \in\left\{1, \ldots, S^{*}\right\}, \\
& T^{-2} \sum_{t=2}^{N} \sum_{S=1-S}^{0}\left(x_{j, S t+s}^{*}\right)^{2} \Rightarrow \frac{1}{2 S}\left[c_{j}^{\top} Q_{1}^{*} c_{j}+\tilde{c}_{j}^{\top} Q_{1}^{*} \tilde{c}_{j}\right], \quad \forall j \in\left\{1, \ldots, S^{*}\right\}, \\
& T^{-2-2 d} \sum_{t=2}^{N} \sum_{s=1-S}^{0} \tilde{x}_{j, S t+s^{*}}^{2} \Rightarrow \frac{1}{S} c_{j}^{\top} Q_{2}^{*} c_{j}, \quad j \in\{0, S / 2\},
\end{aligned}
$$




$$
\begin{aligned}
& T^{-2-2 d} \sum_{t=2}^{N} \sum_{S=1-S}^{0}\left(\tilde{x}_{j, S t+s}^{* *}\right)^{2} \Rightarrow \frac{1}{2 S}\left[c_{j}^{\top} Q_{2}^{*} c_{j}+\tilde{c}_{j}^{\top} Q_{2}^{*} \tilde{c}_{j}\right], \quad \forall j \in\left\{1, \ldots, S^{*}\right\}, \\
& T^{-2-2 d} \sum_{t=2}^{N} \sum_{s=1-S}^{0}\left(\tilde{x}_{j, S t+s}^{*}\right)^{2} \Rightarrow \frac{1}{2 S}\left[c_{j}^{\top} Q_{2}^{*} c_{j}+\tilde{c}_{j}^{\top} Q_{2}^{*} \tilde{c}_{j}\right], \quad \forall j \in\left\{1, \ldots, S^{*}\right\} .
\end{aligned}
$$

After obtaining the convergence results above, the asymptotic null distributions of the wild bootstrap FSVR statistics given in the first part of this theorem can be obtained straightforwardly.

Now, we switch to the proof of the second part of the theorem. Firstly, consider the non-parametric wild bootstrap $\tau_{0}^{*}(d)$ statistic. From the result $\tau_{0}^{*}(d) \Rightarrow \xi_{\eta, 0}(d)$ obtained in the first part of the theorem, we can conclude that, uniformly in probability, $G_{0, T}^{*}(.) \rightarrow G_{0}($.$) , where G_{0}($.$) is the CDF of \xi_{\eta, 0}(d)$. This result coupled with the proof of Corollary 1 in Hansen (2000) implies that $P_{T}^{*}$ converges weakly to $U[0,1]$ under the conditions of Theorem 2. The corresponding results for the other FSVR statistics can be established similarly.

\section{Appendix B: Simulation and empirical results}

See Tables 1, 2, 3, 4, 5, 6, 7, 8, 9, 10, 11, 12, 13, 14 and 15. 


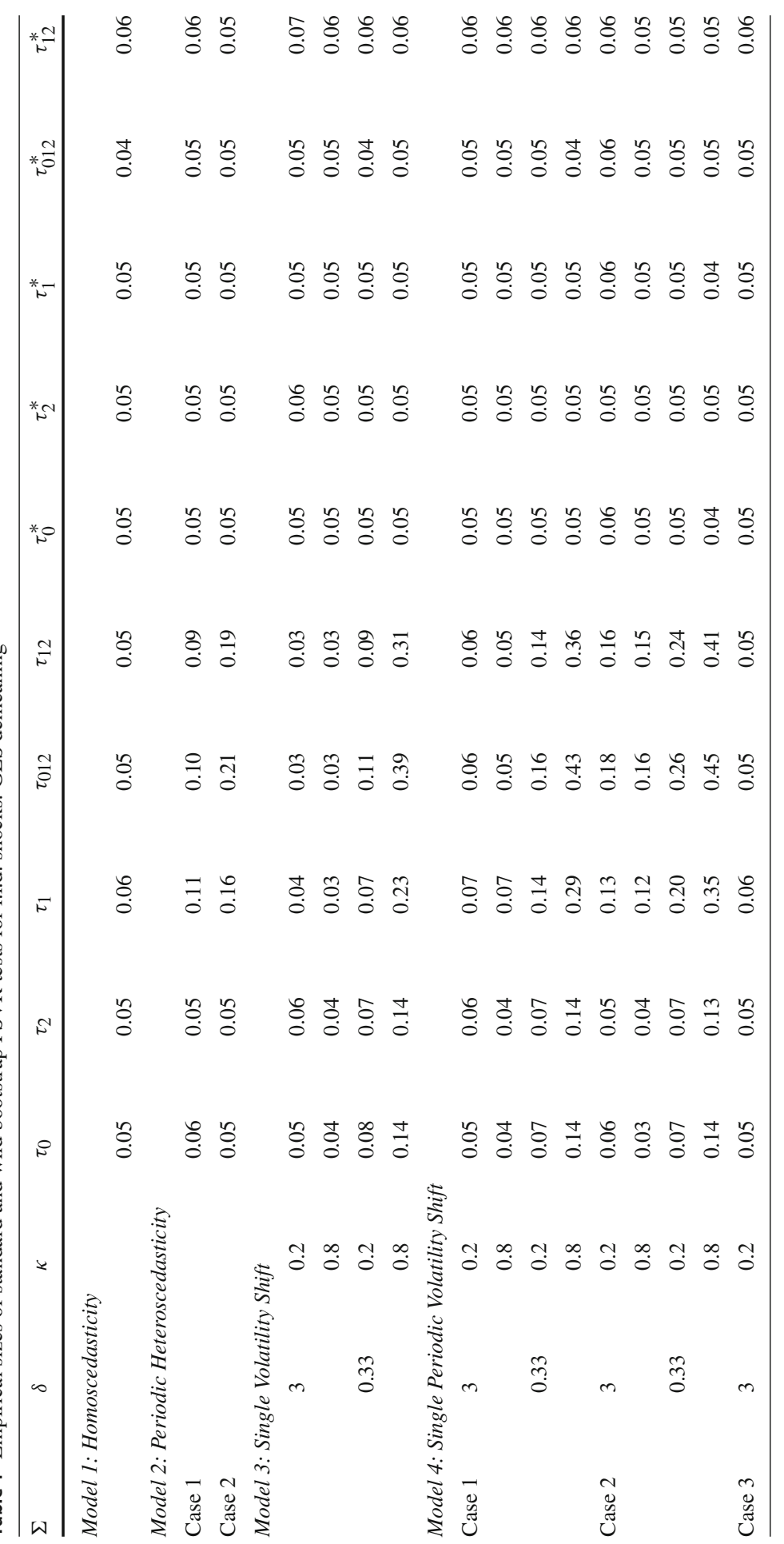




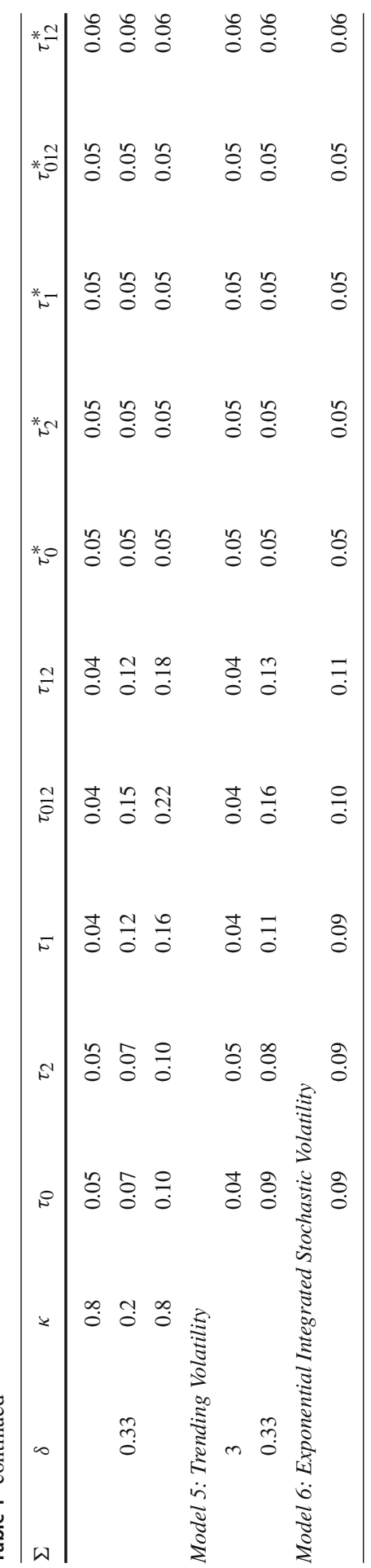




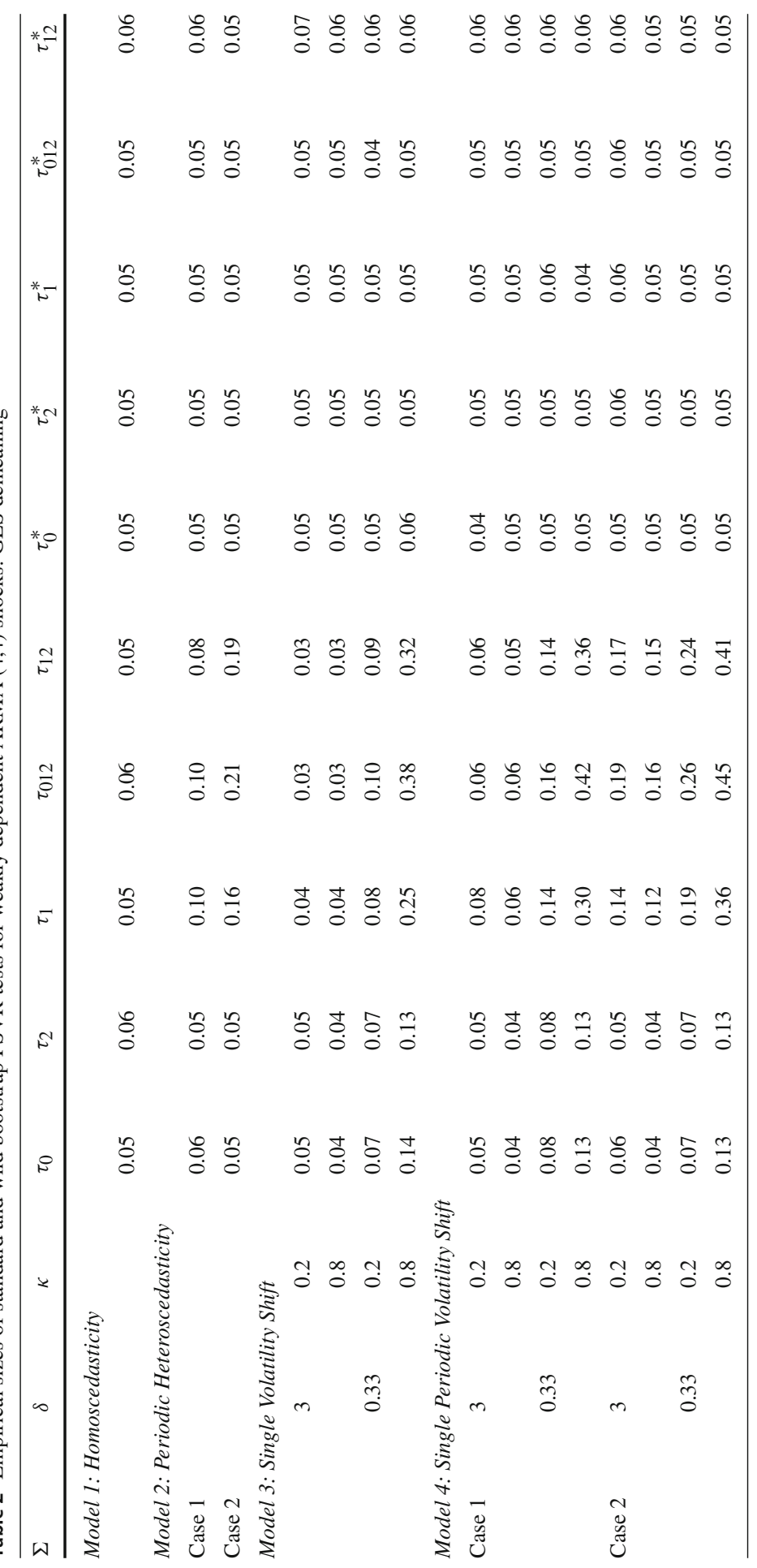




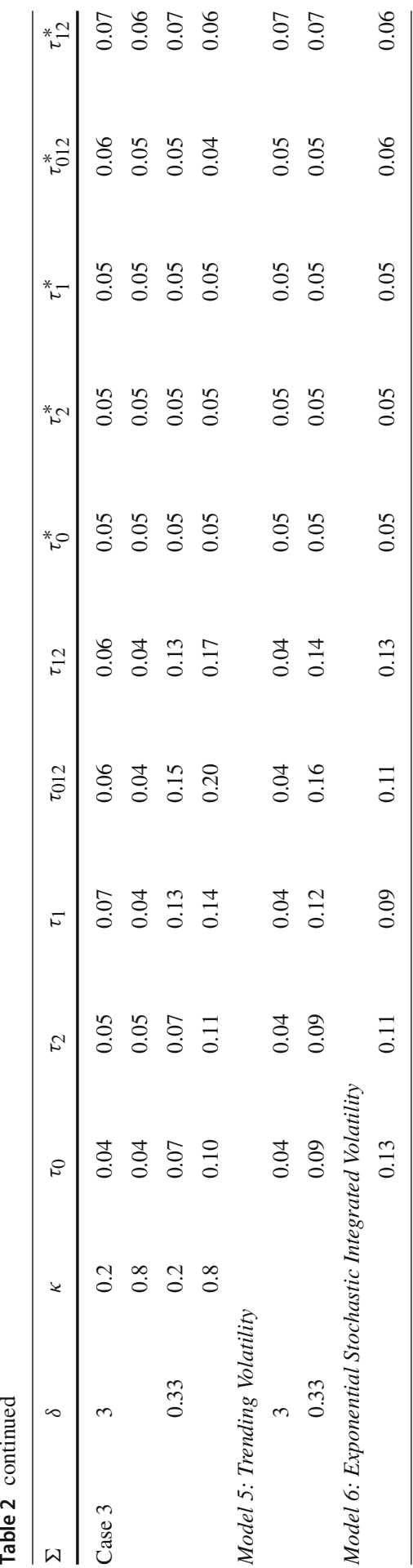




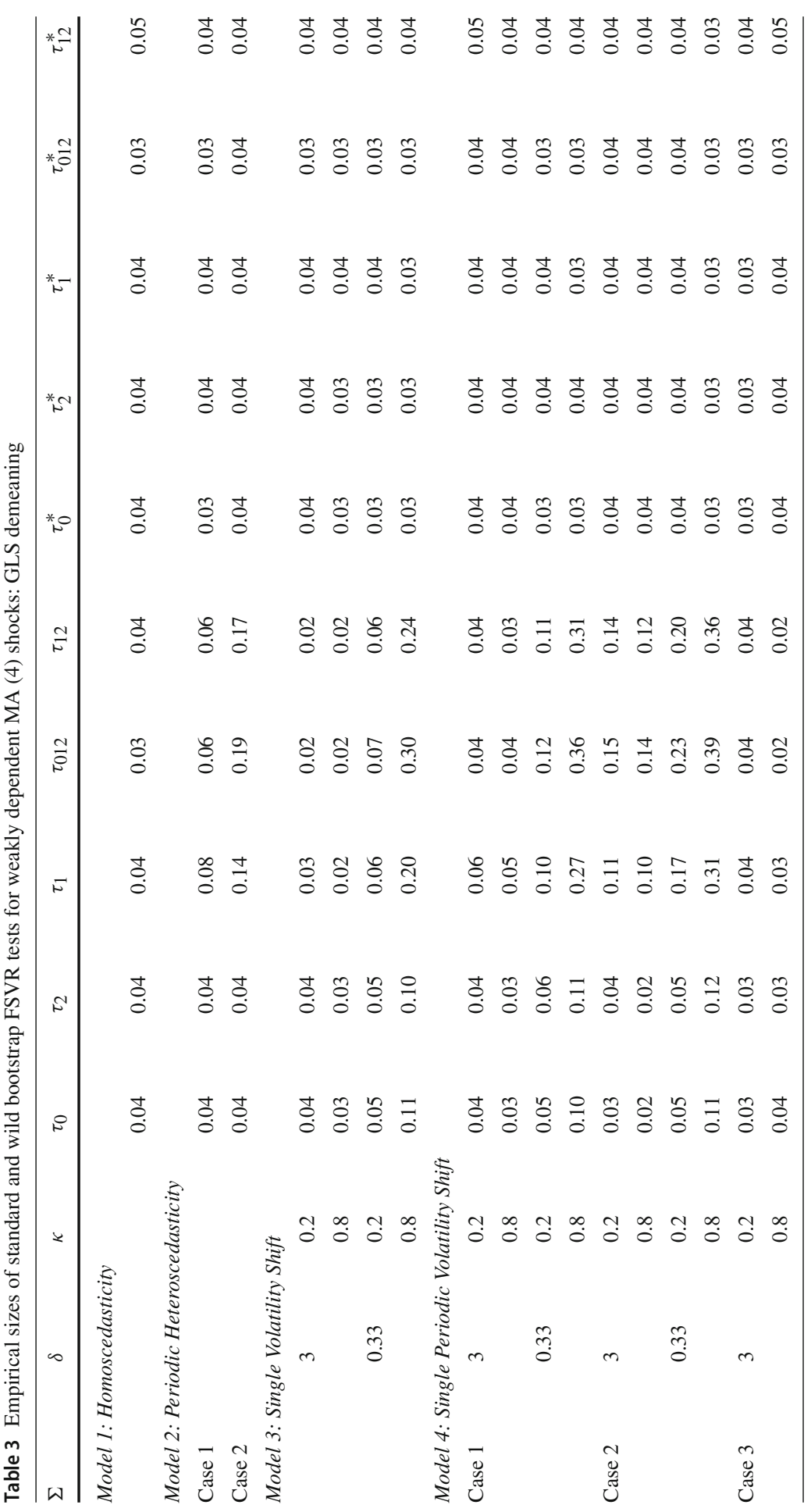




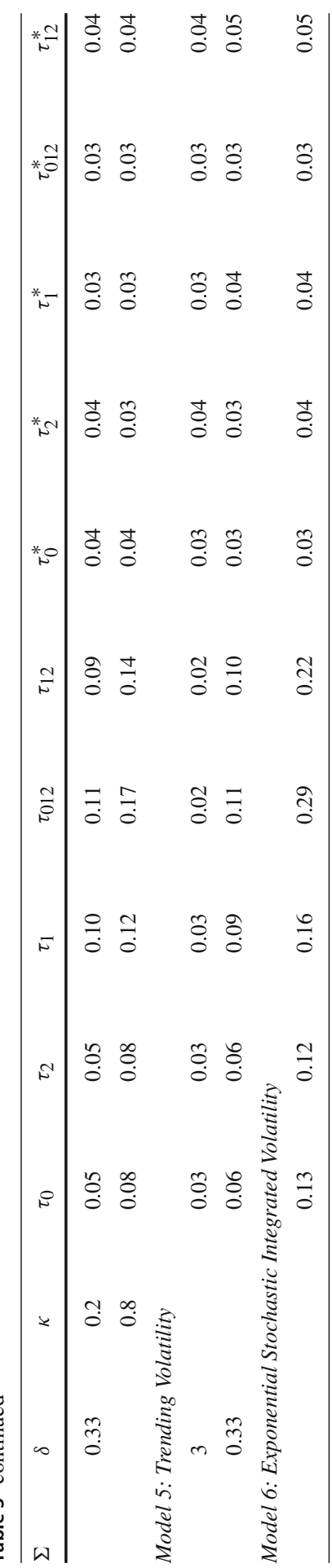




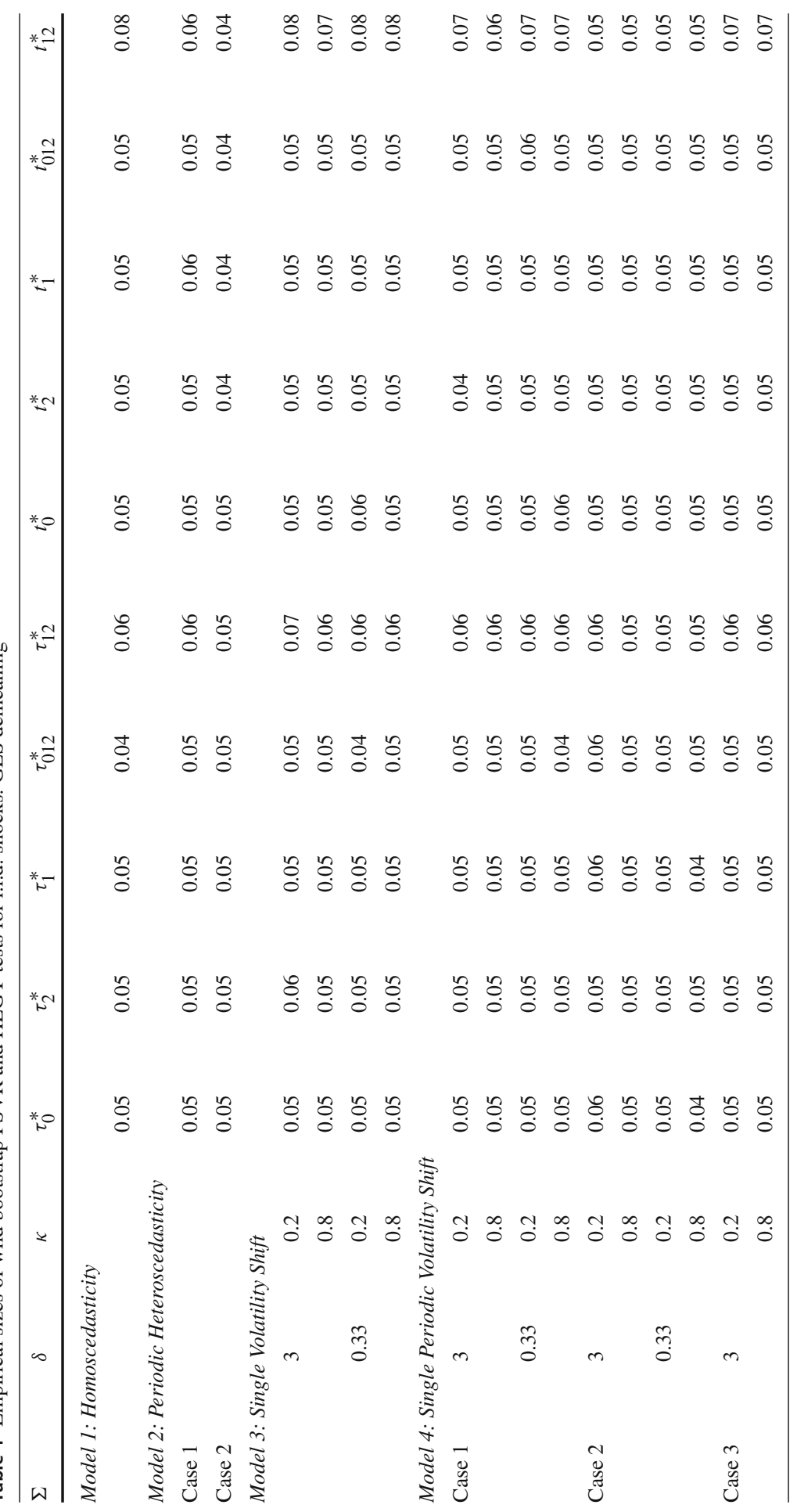




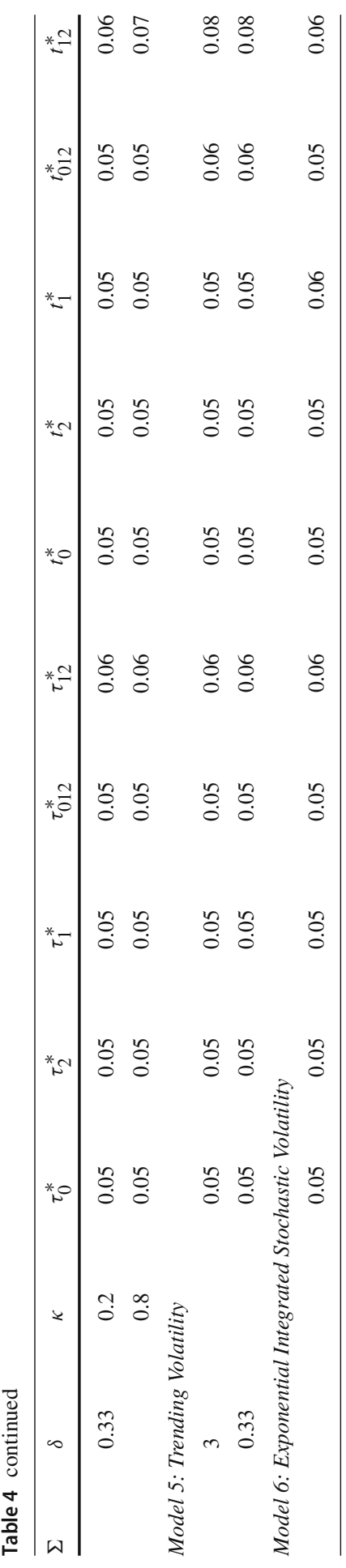




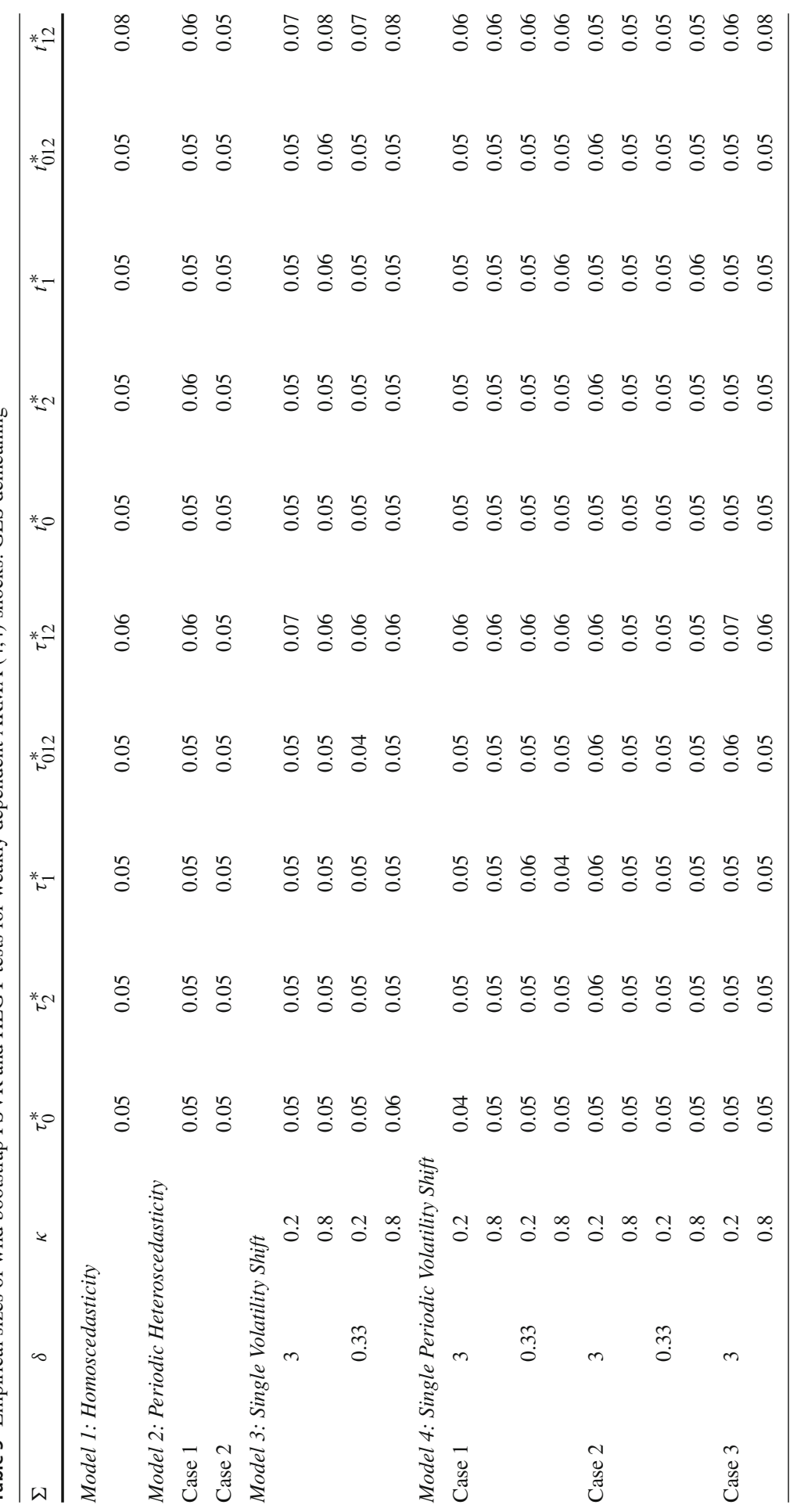




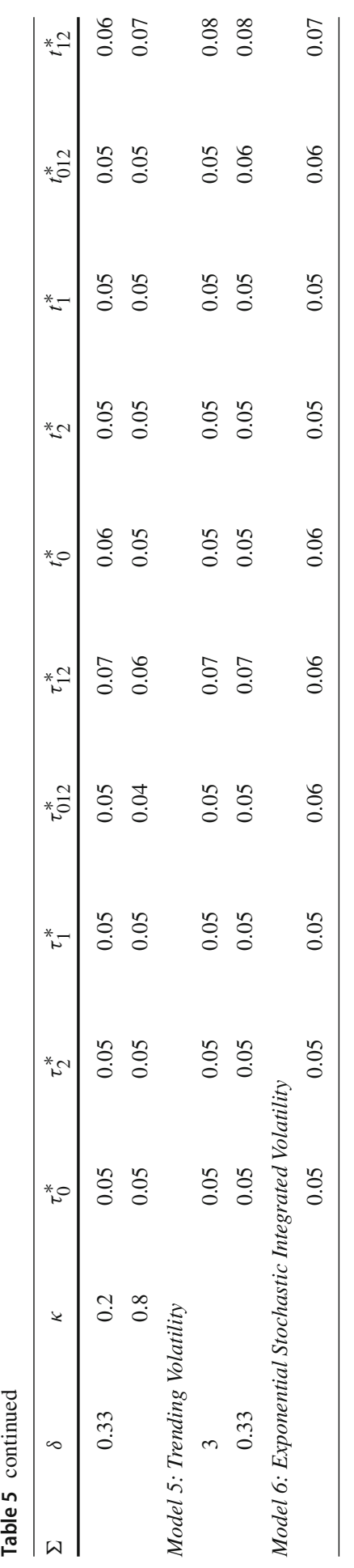




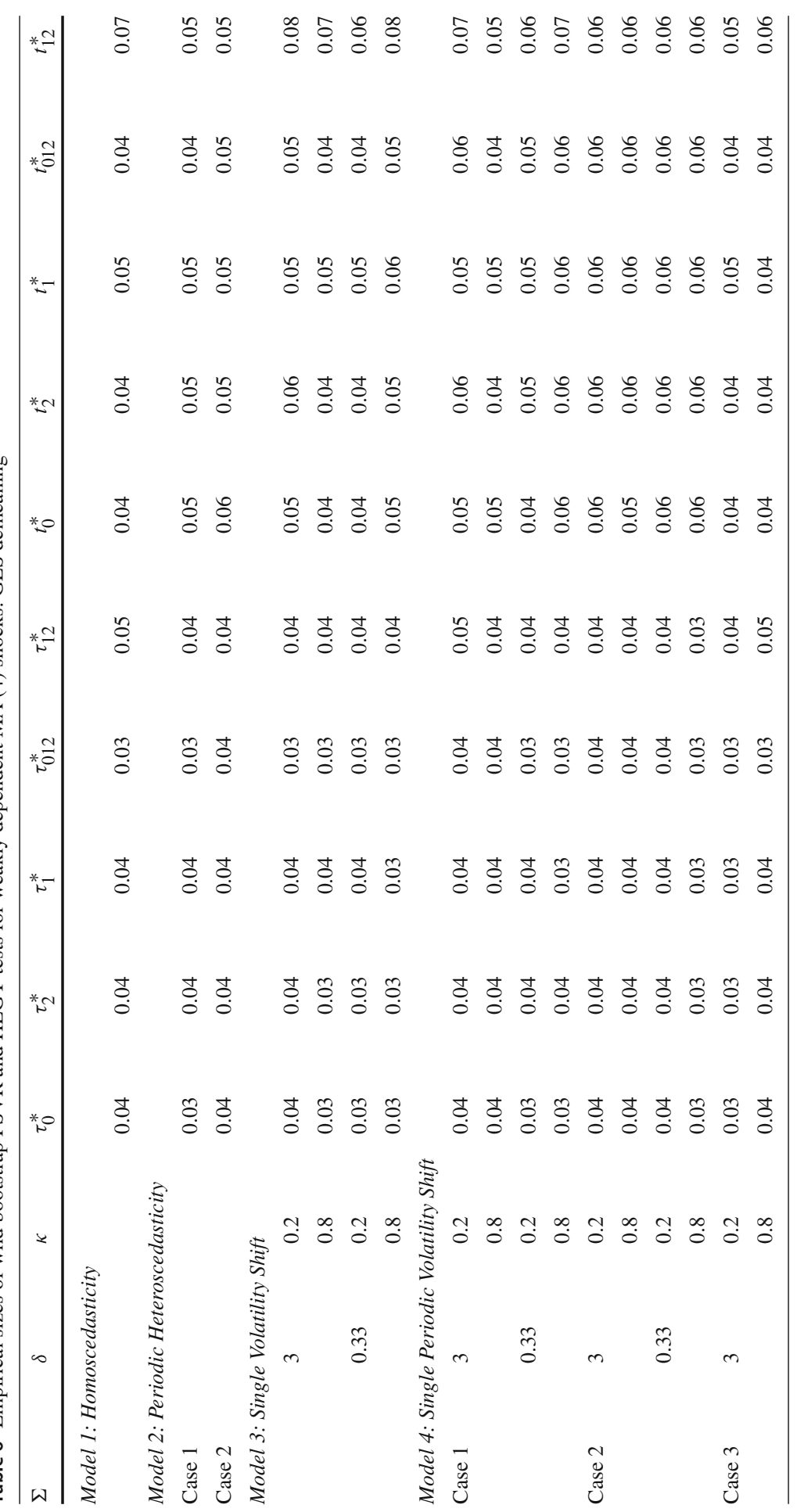




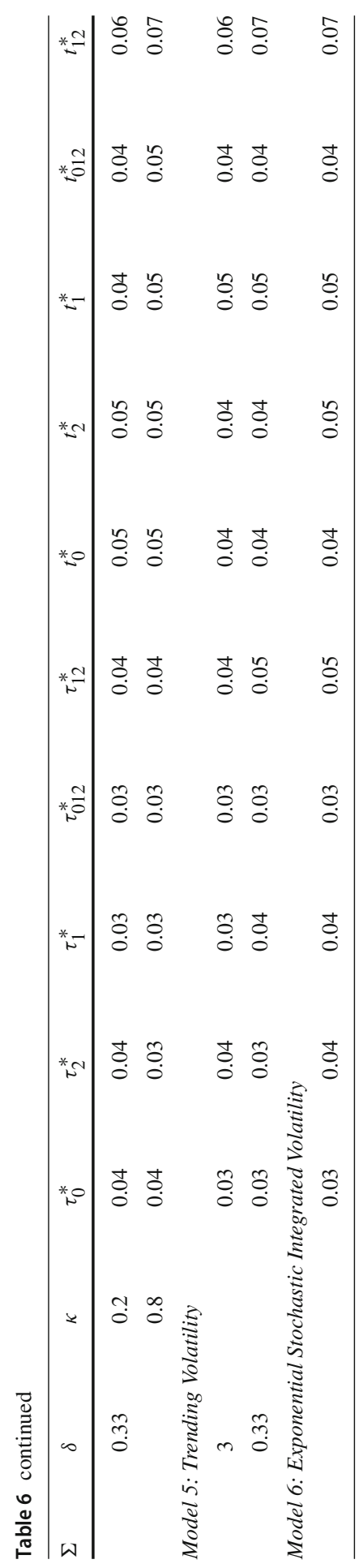




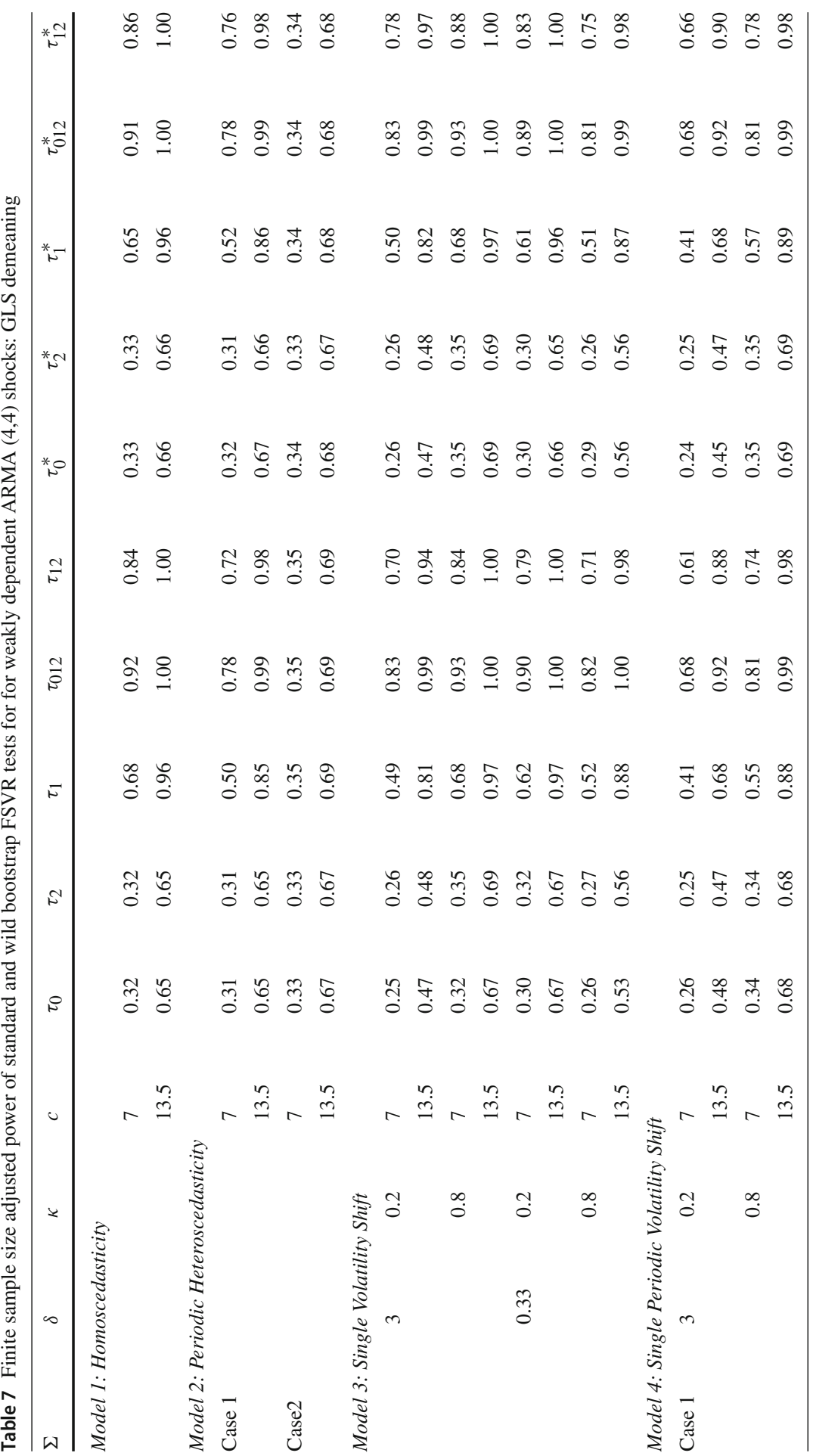




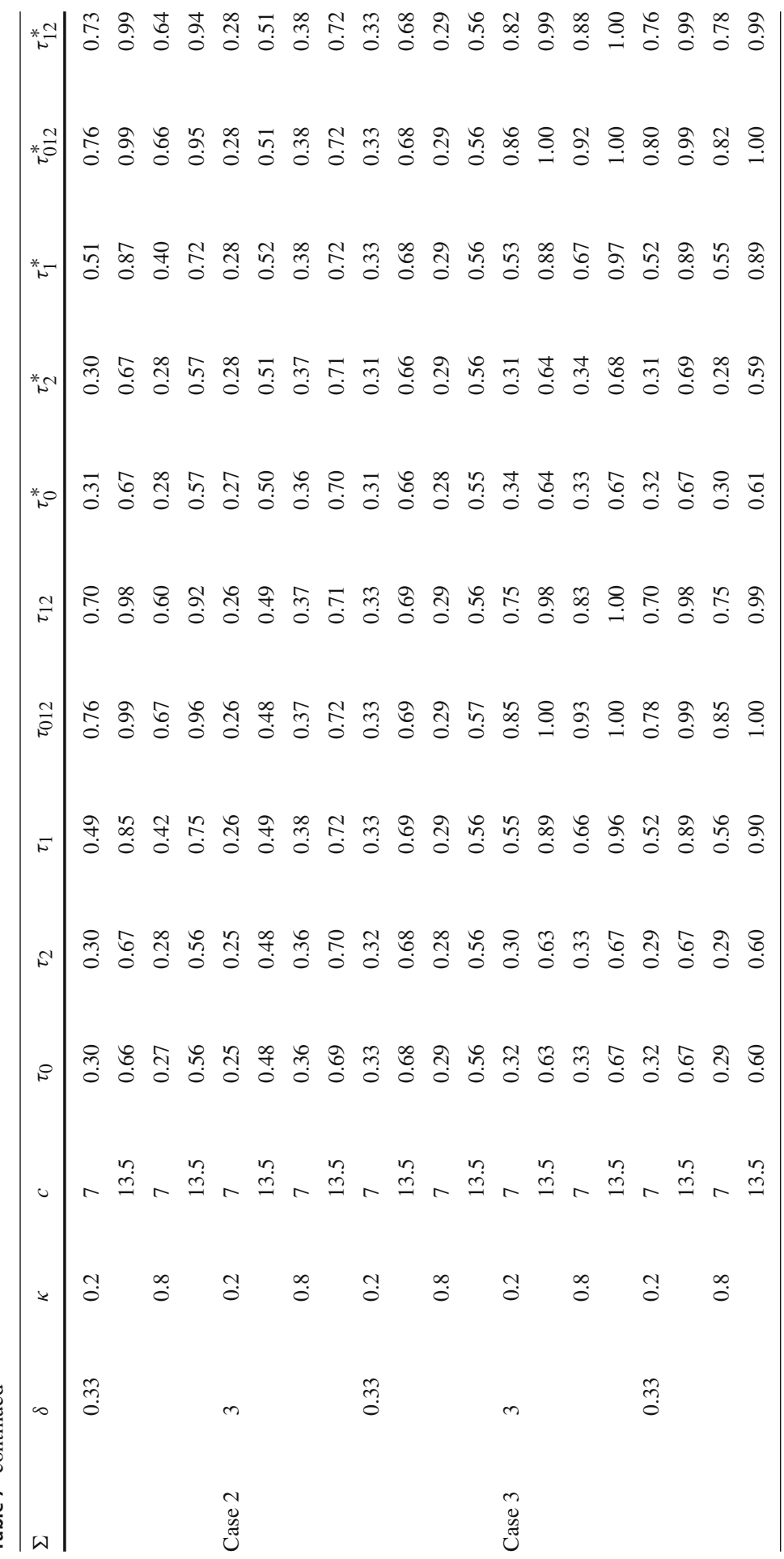




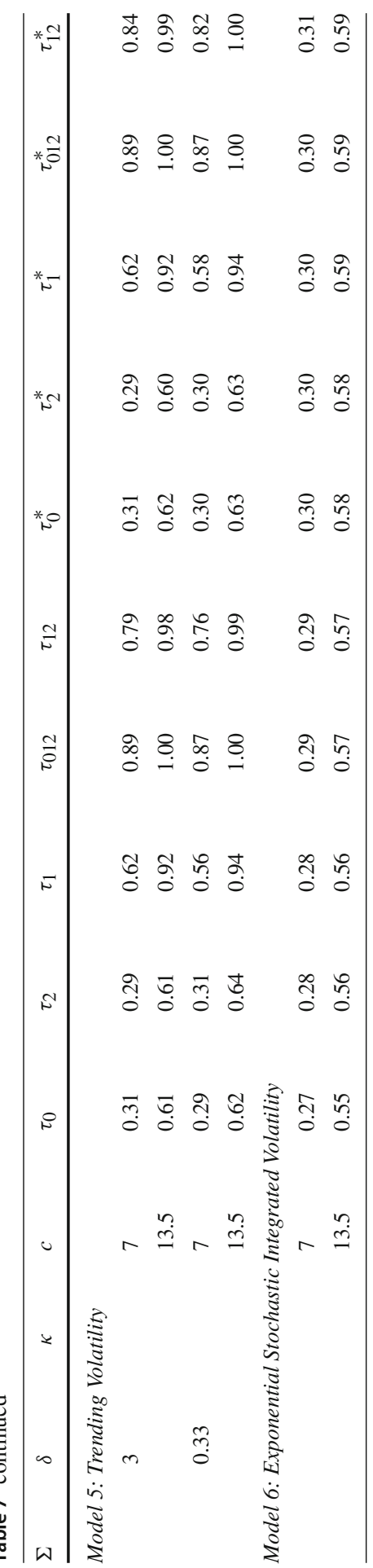




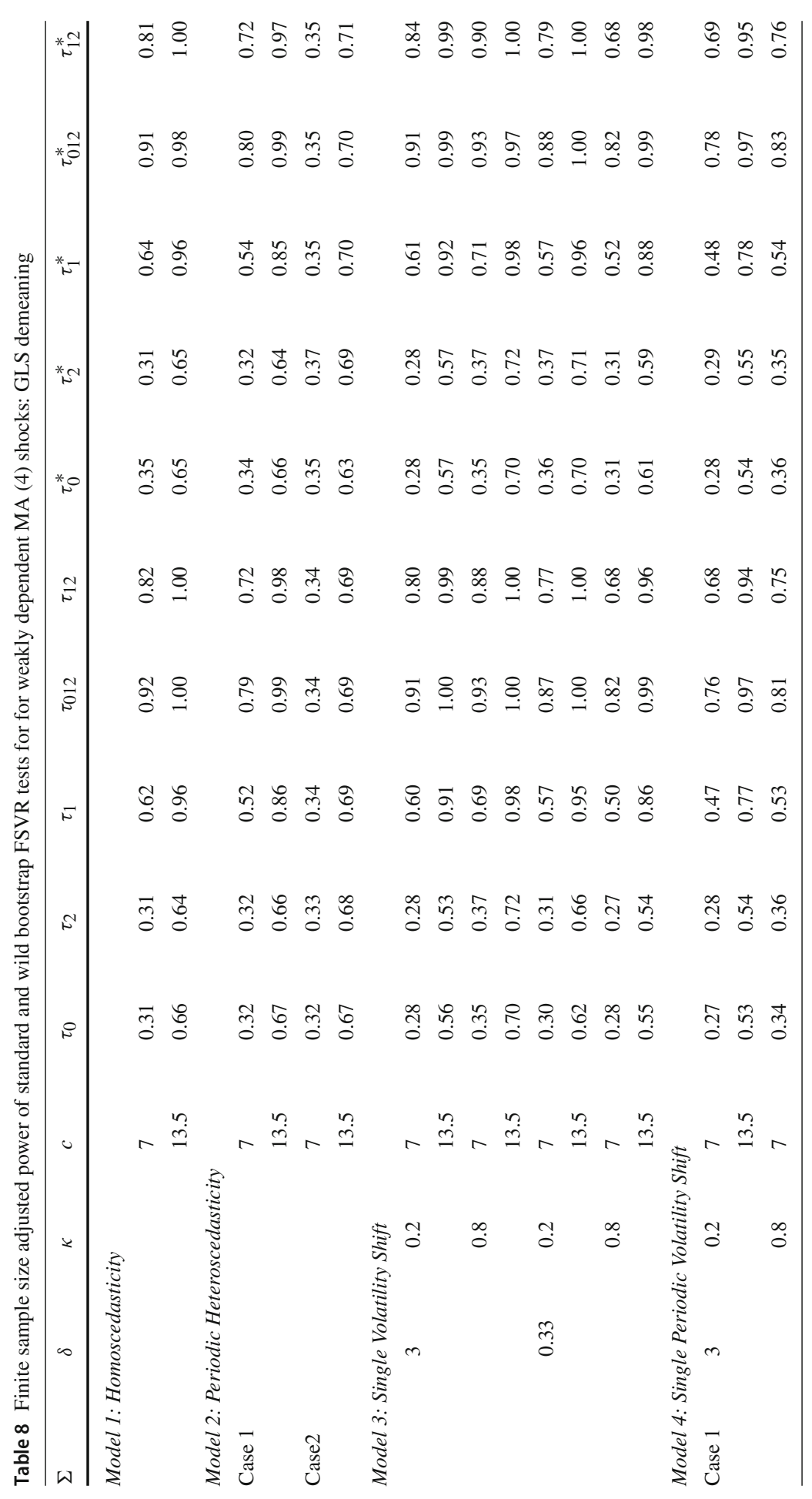




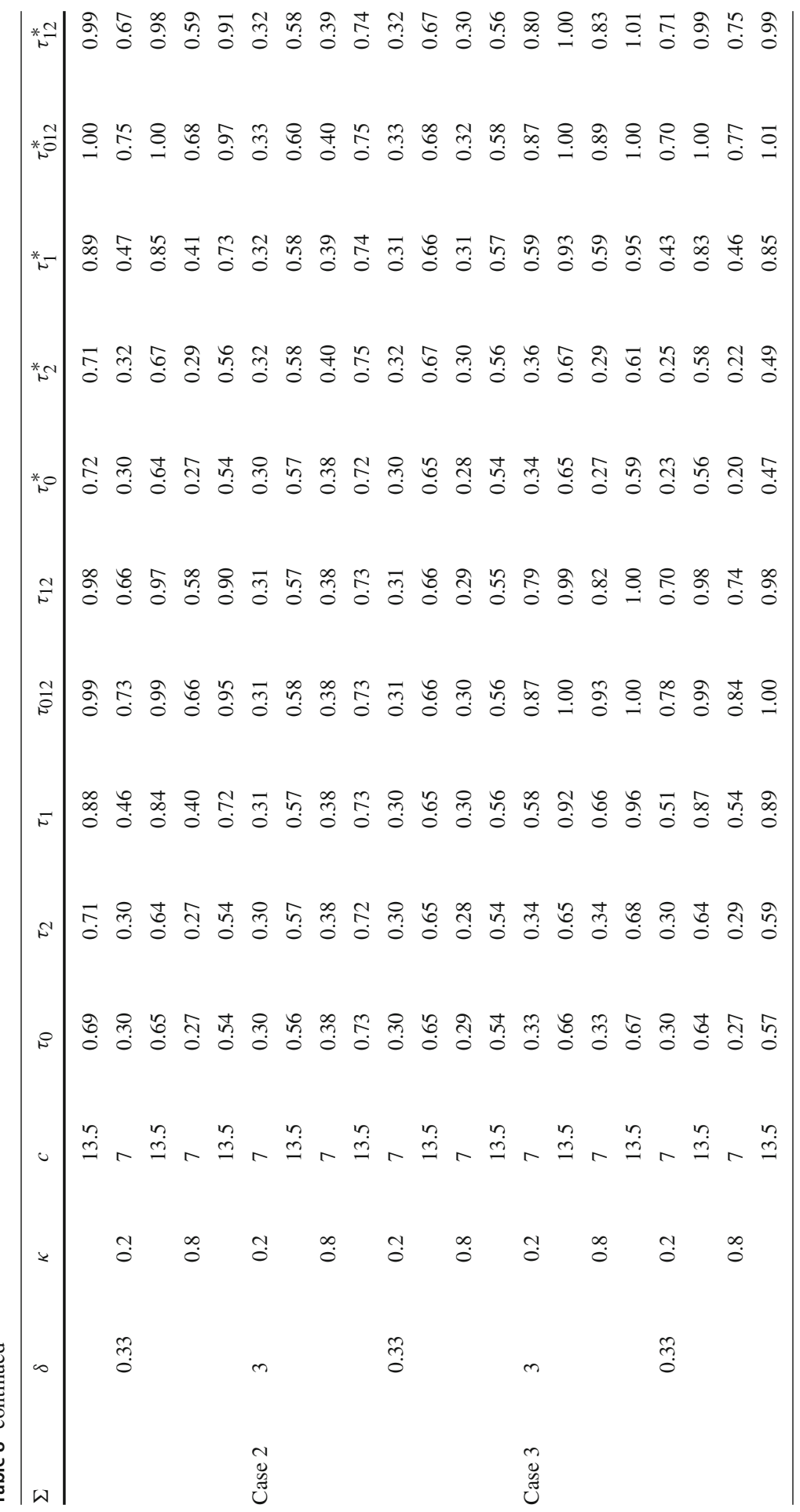




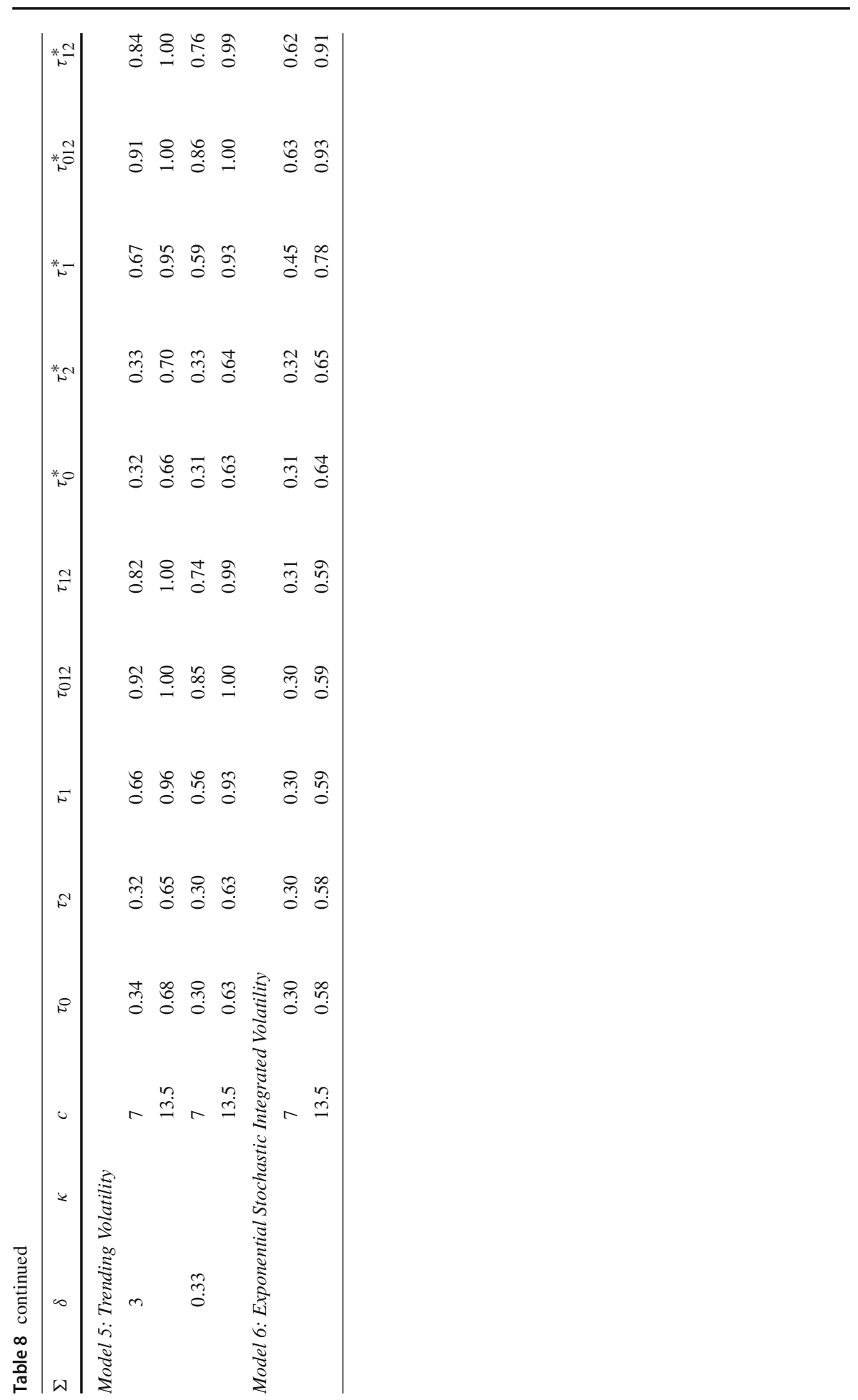




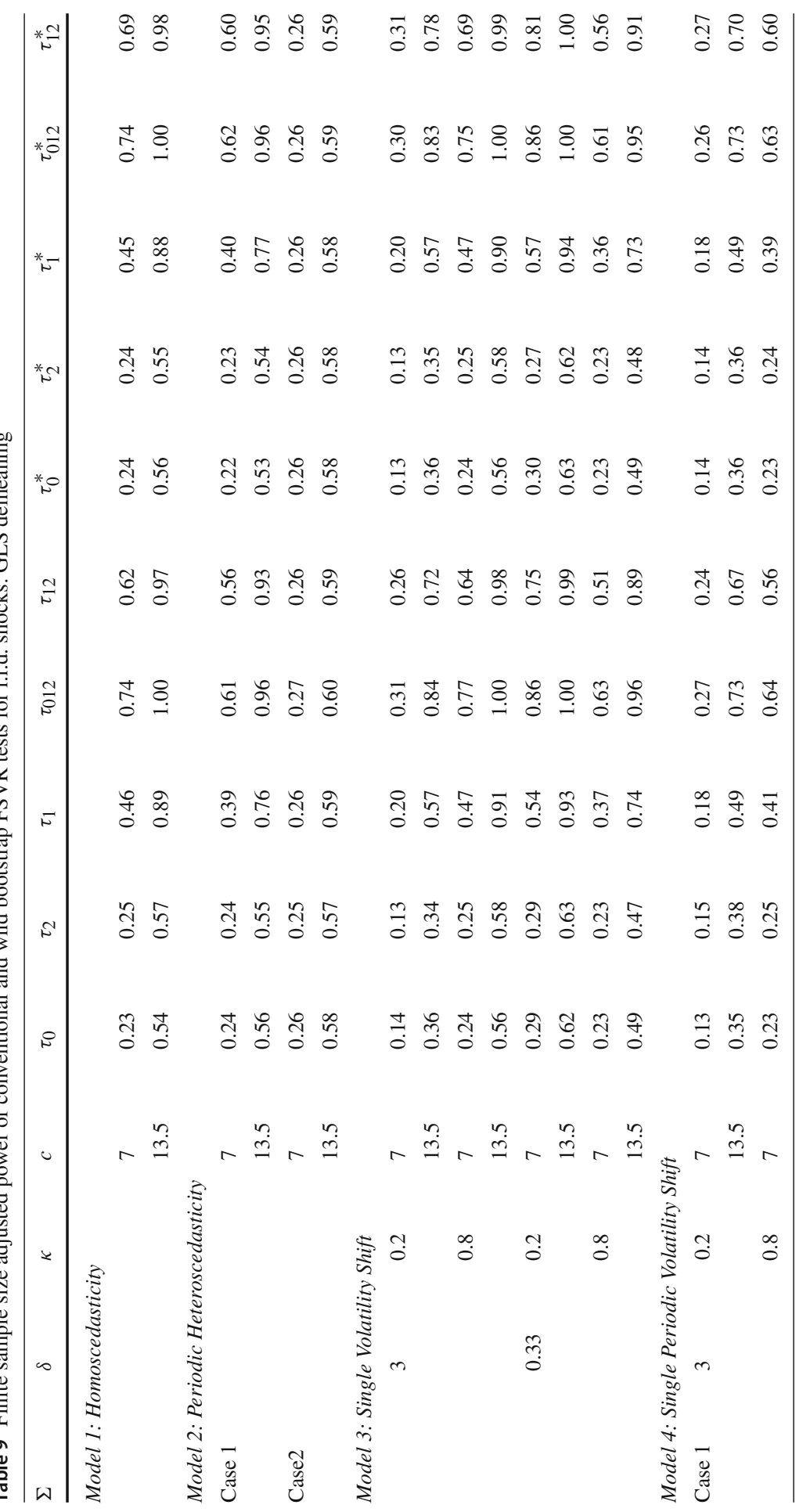




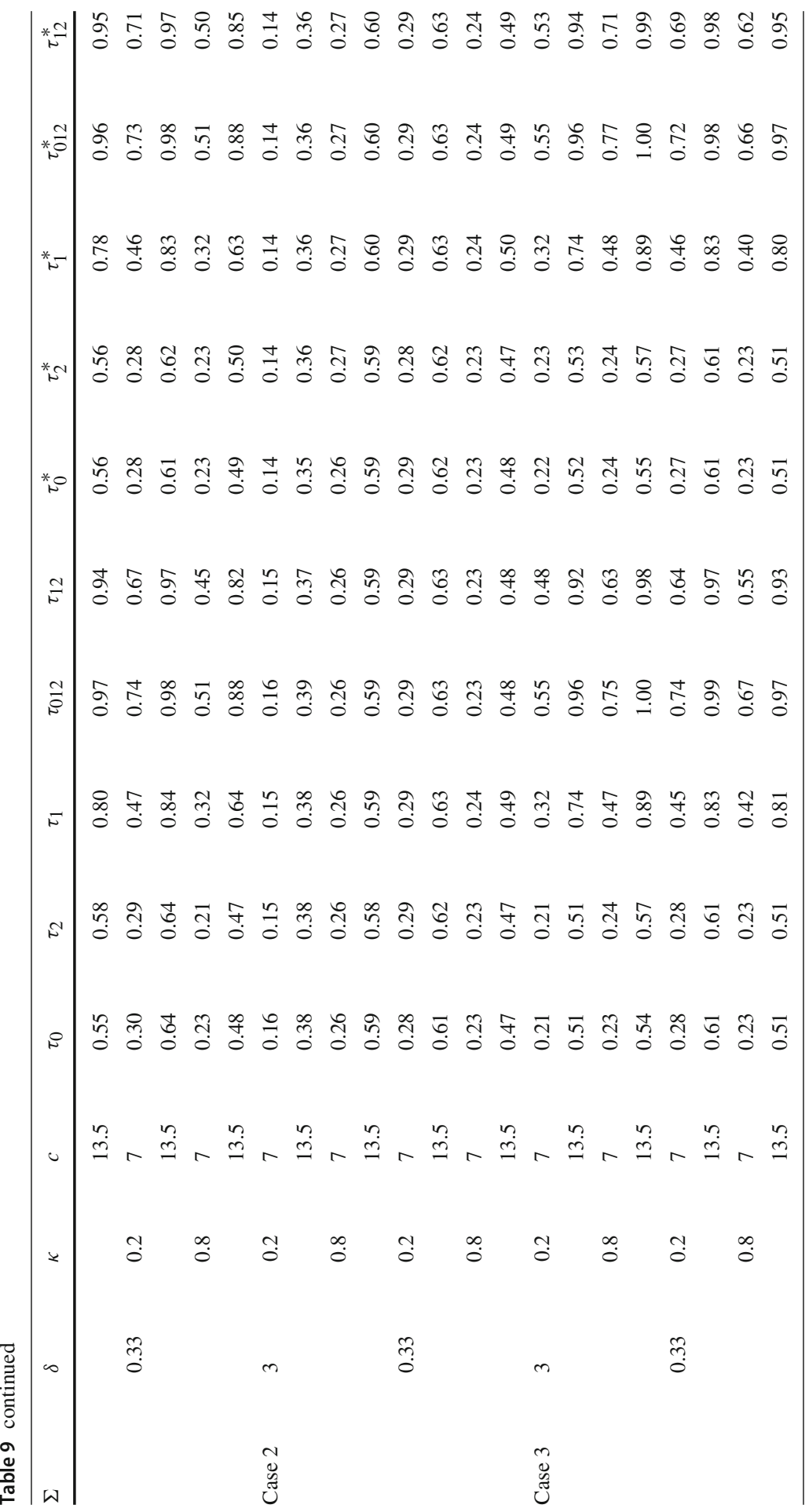




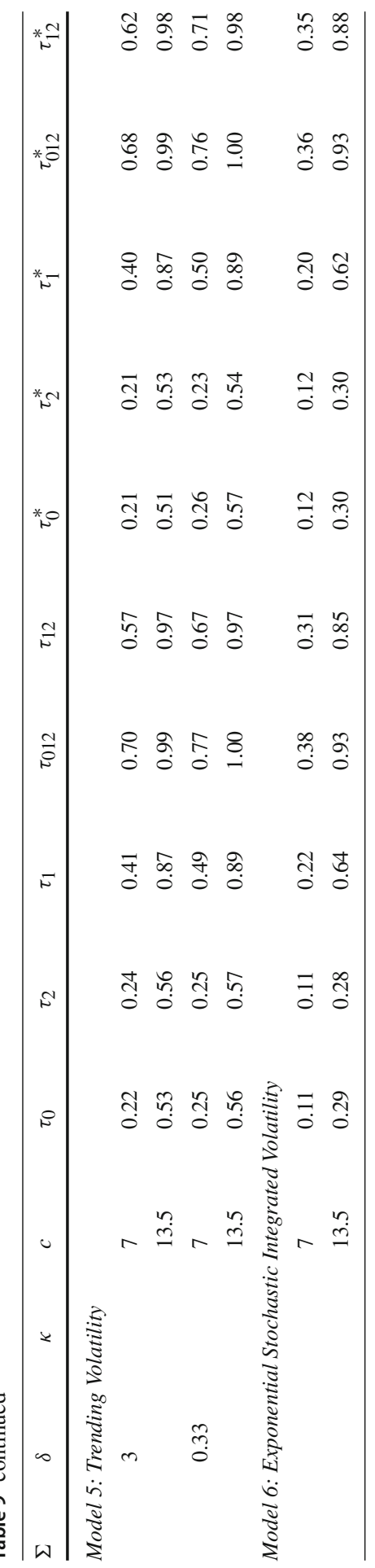




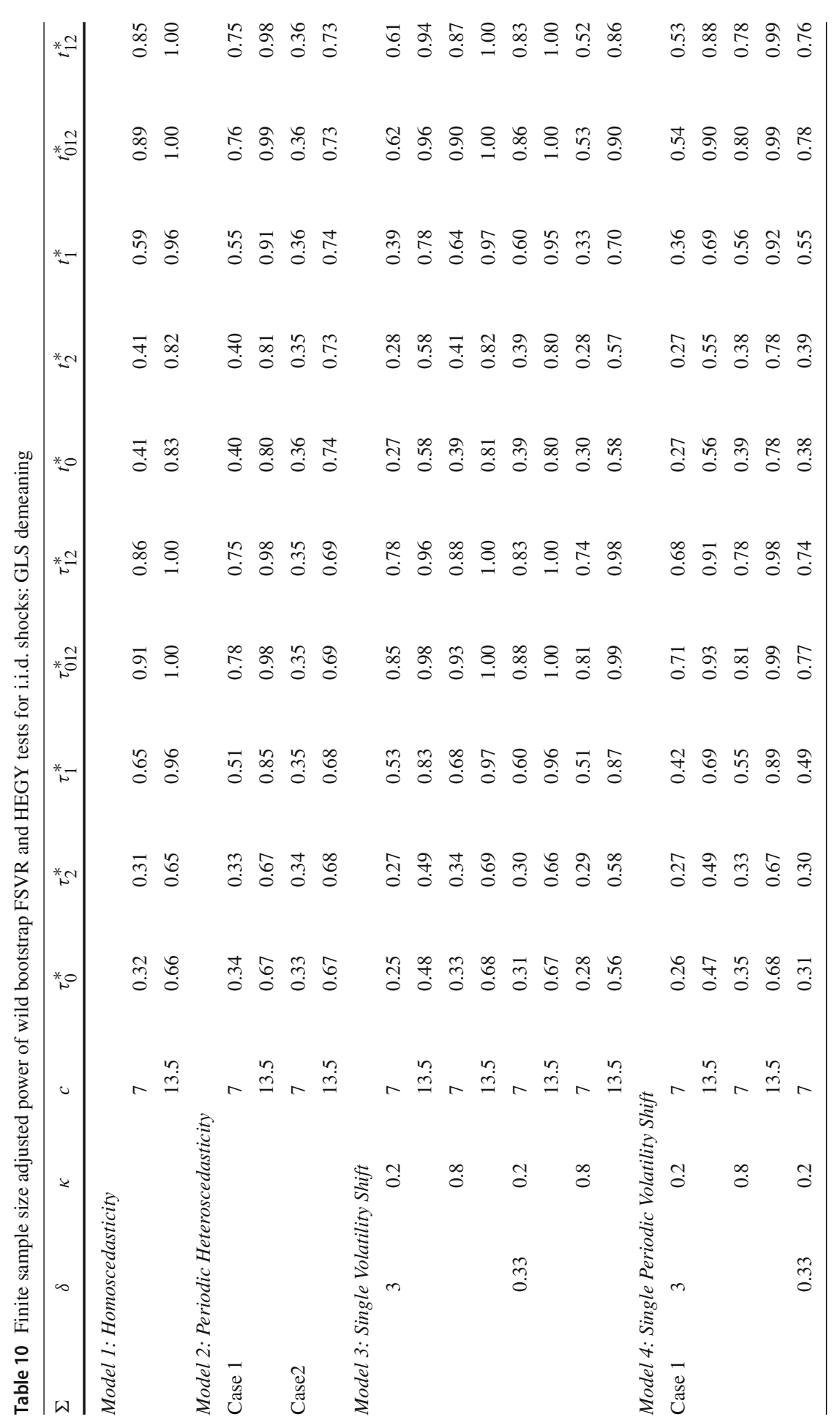




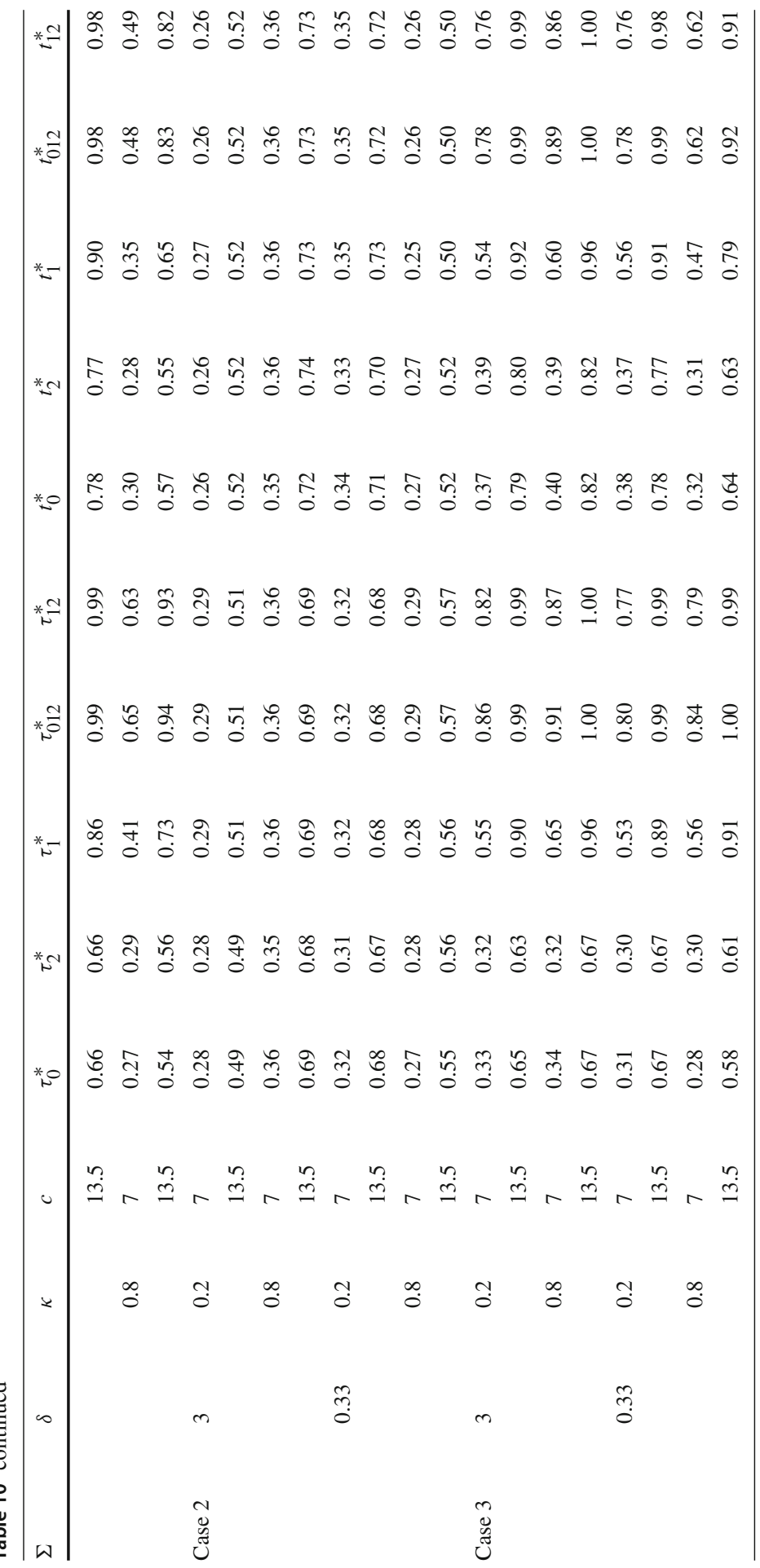




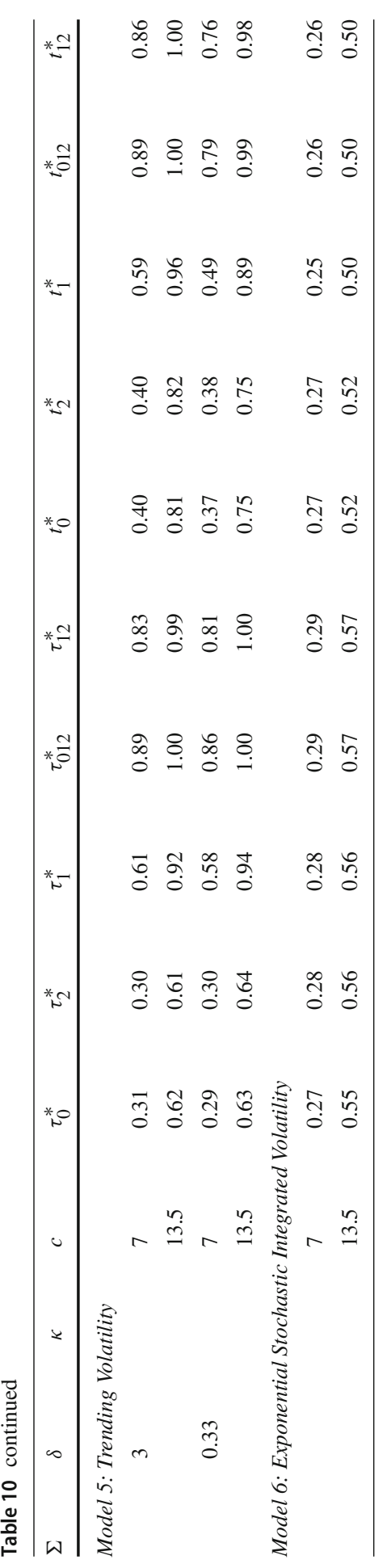




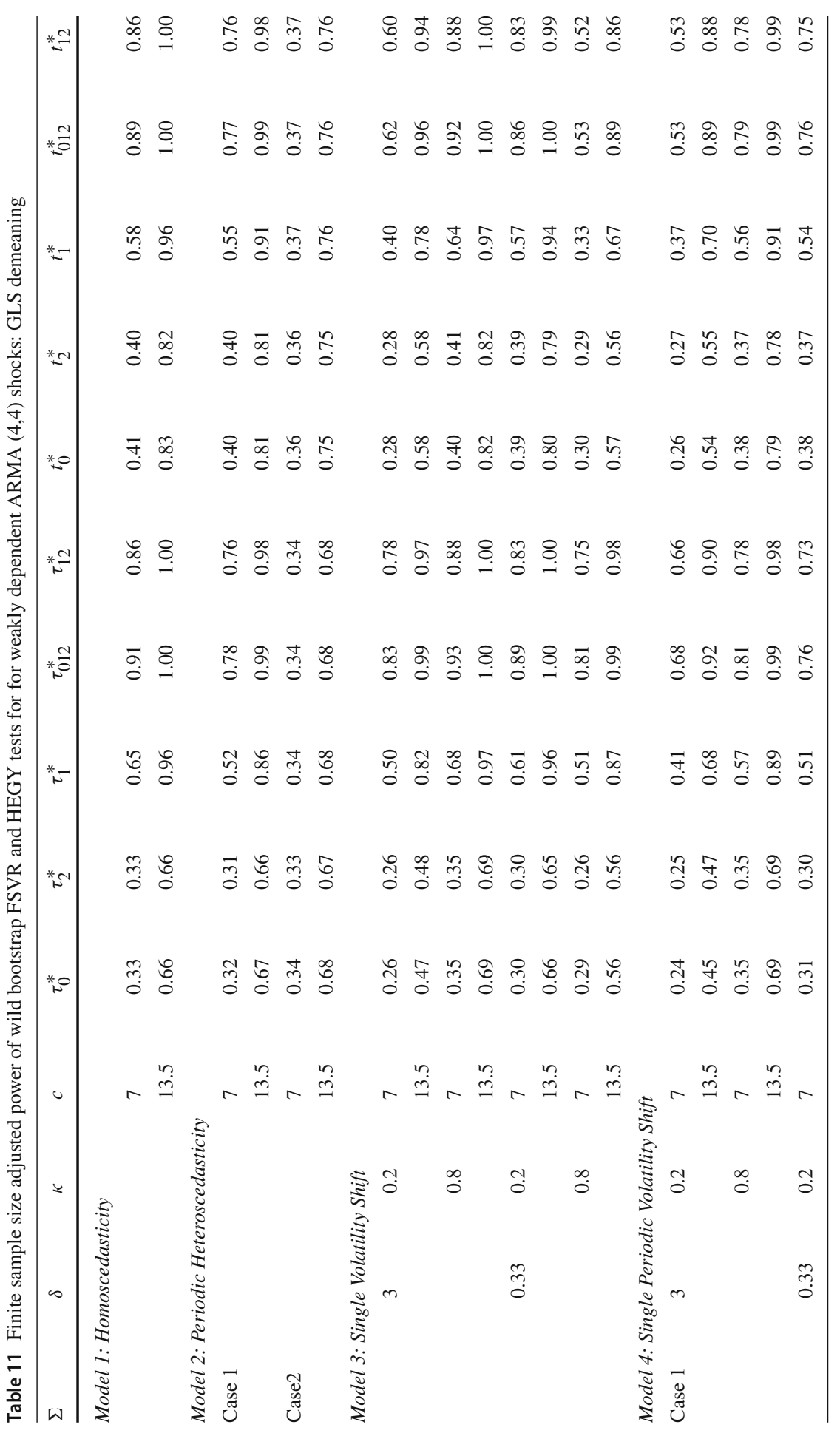




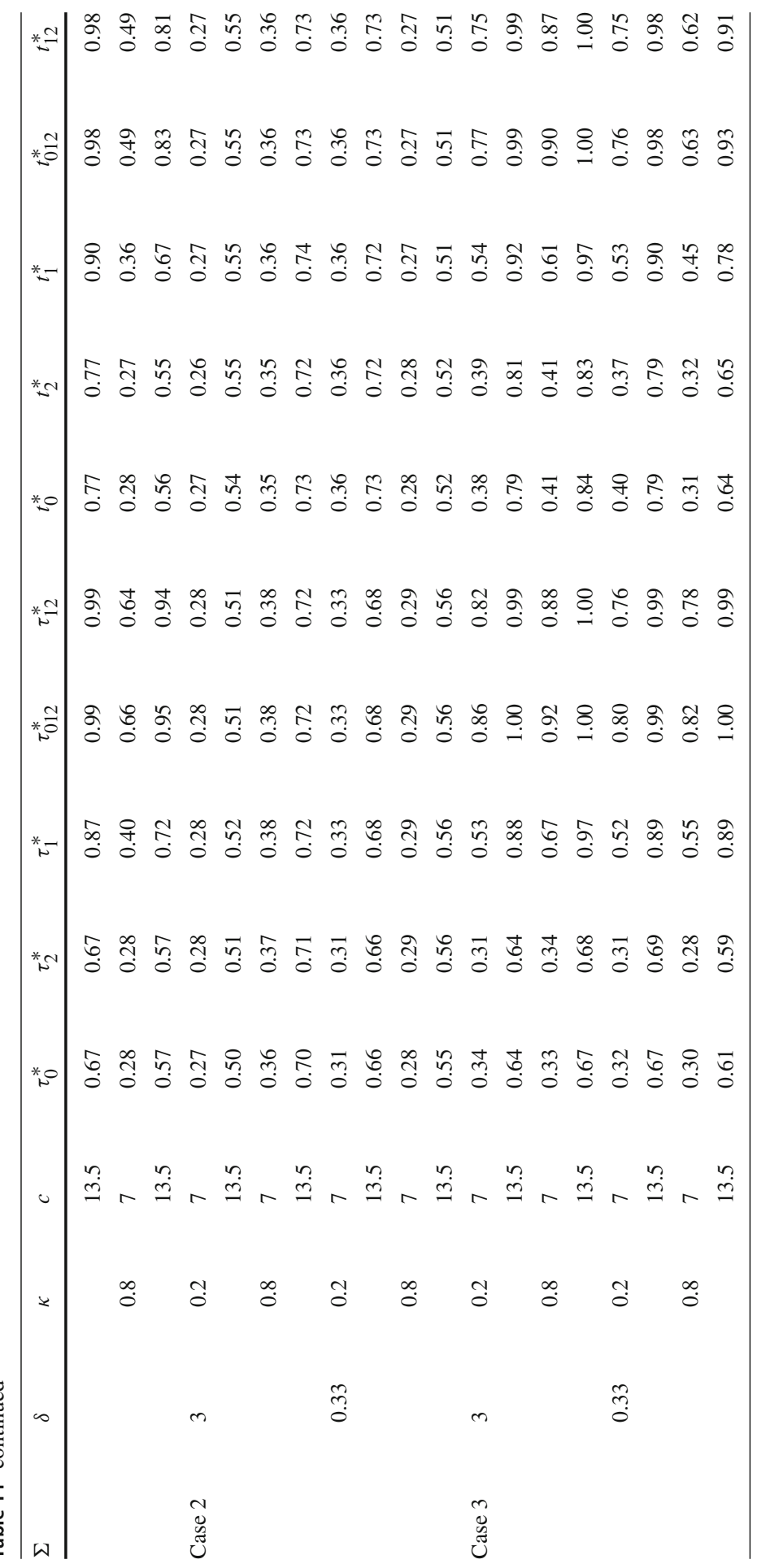




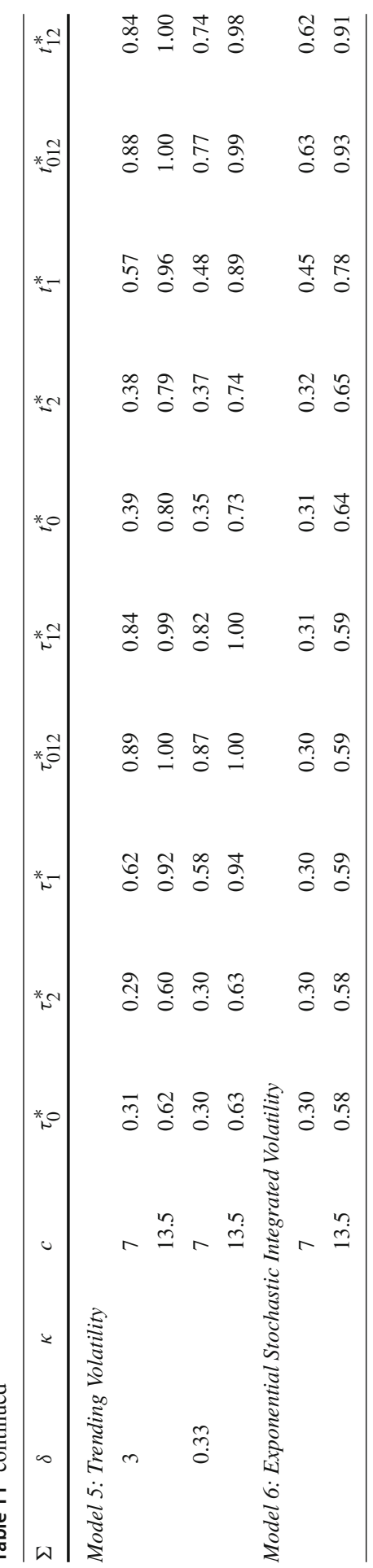




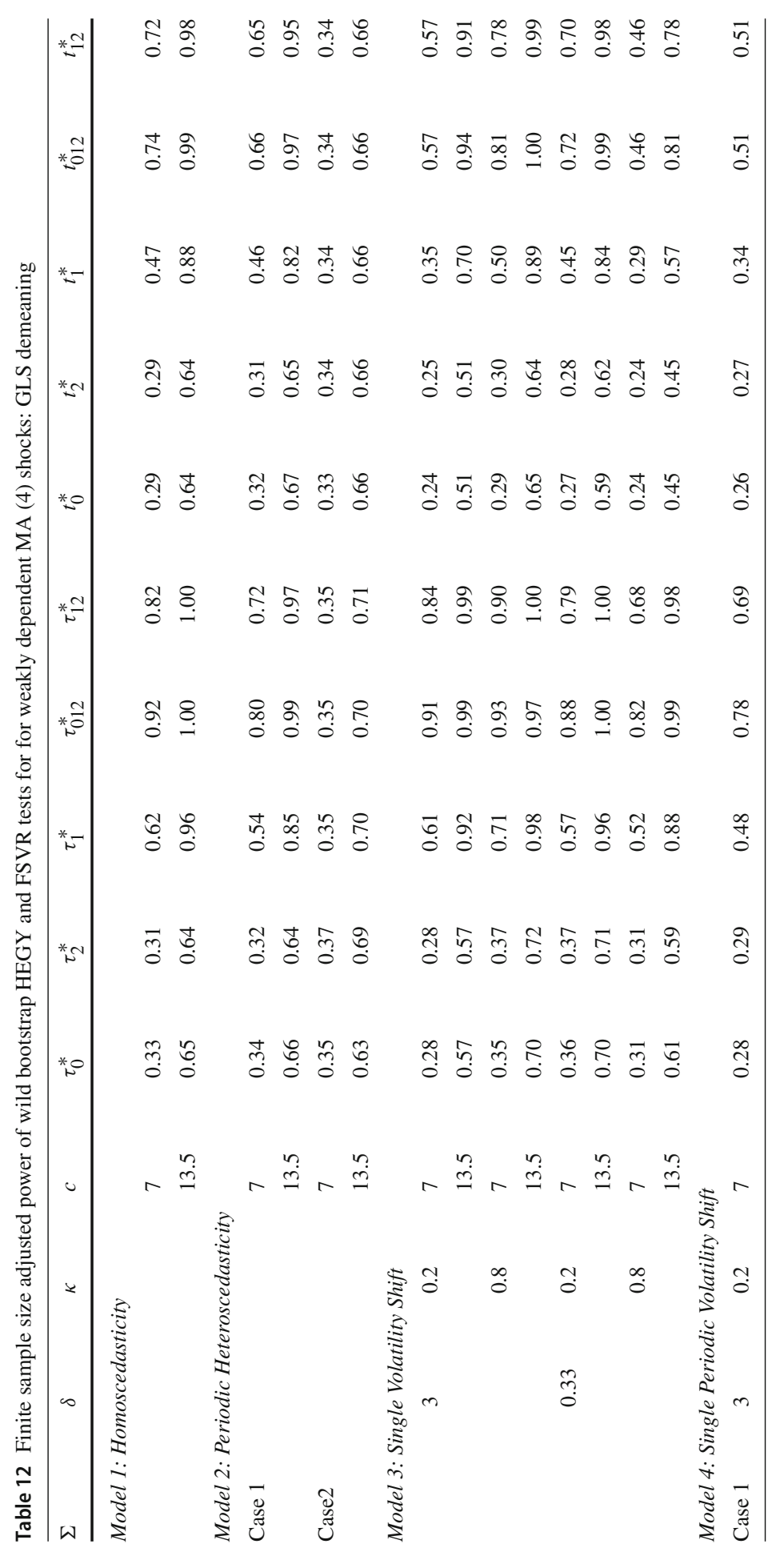




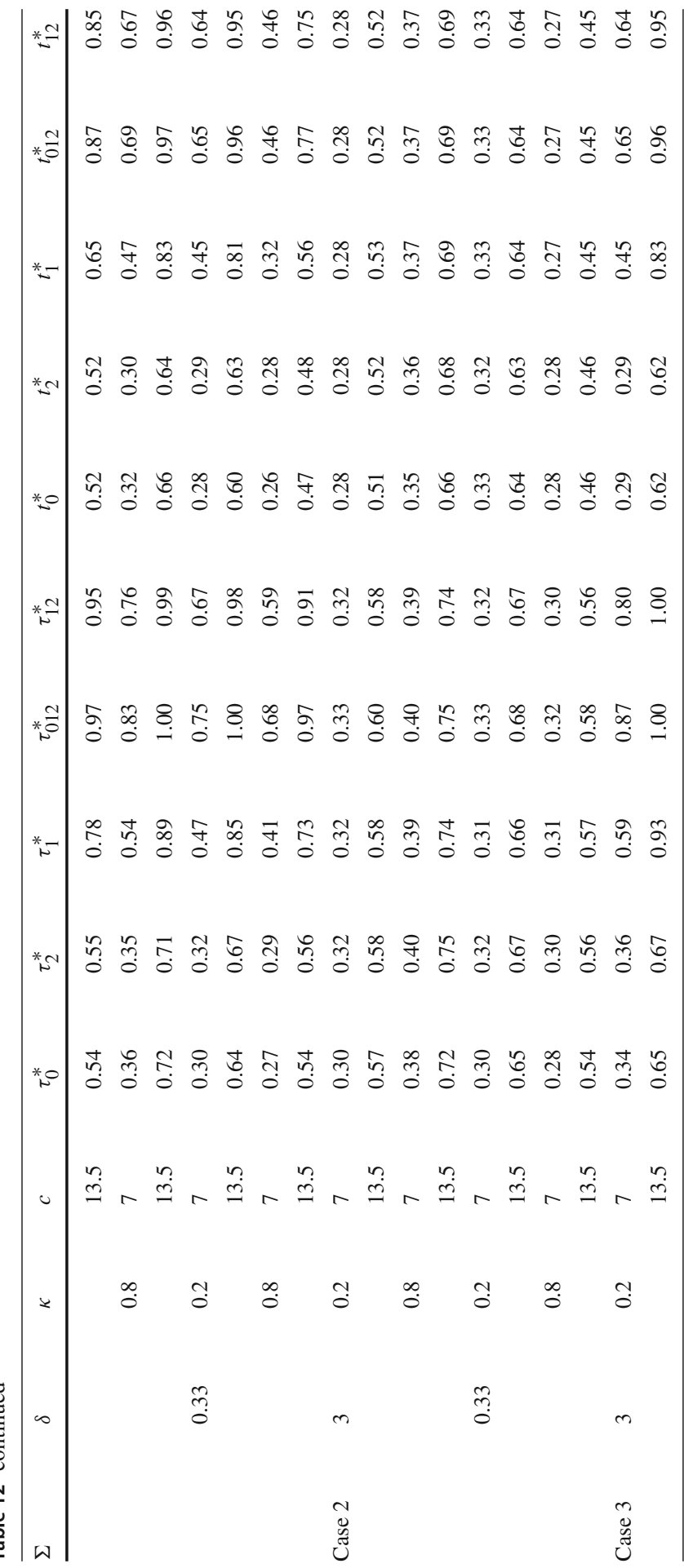




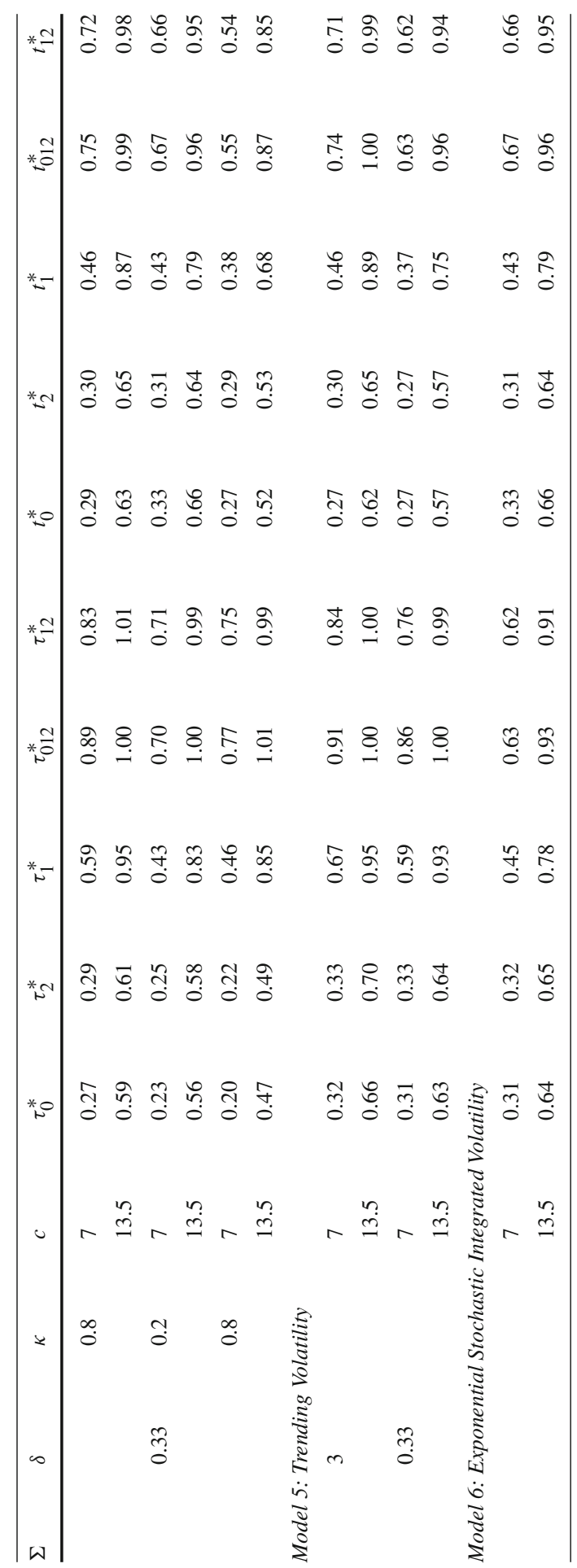


Table 13 P-values of the Wild-bootstrap FSVR and HEGY tests on the U.S. Unemployment Data

\begin{tabular}{llllllllll}
\hline Frequency & 0 & $\pi$ & $\pi / 6$ & $\pi / 3$ & $\pi / 2$ & $2 \pi / 3$ & $5 \pi / 6$ & $0, \ldots, \pi$ & $1, \ldots, \pi$ \\
\hline FSVR & $\mathbf{0 . 0 7 0}$ & 0.000 & $\mathbf{0 . 9 9 9}$ & $\mathbf{0 . 9 9 9}$ & $\mathbf{0 . 9 9 9}$ & $\mathbf{0 . 9 9 9}$ & $\mathbf{0 . 9 9 9}$ & 0.000 & 0.000 \\
HEGY & 0.001 & $\mathbf{0 . 3 0 8}$ & $\mathbf{0 . 9 9 9}$ & $\mathbf{1 . 0 0 0}$ & $\mathbf{1 . 0 0 0}$ & $\mathbf{0 . 9 9 9}$ & $\mathbf{0 . 9 9 9}$ & 0.033 & $\mathbf{0 . 2 2 6}$ \\
\hline
\end{tabular}

Significance levels of the HEGY and FSCR tests are 0.05

Table 14 Empirical size of wild bootstrap FSVR seasonal unit root tests for $S=12$ : GLS demeaning

\begin{tabular}{|c|c|c|c|c|c|}
\hline Serial Correlation & $\tau_{0}^{*}$ & $\tau_{2}^{*}$ & $\tau_{1}^{*}$ & $\tau_{012}^{*}$ & $\tau_{12}^{*}$ \\
\hline \multicolumn{6}{|c|}{ Model 1: Homoscedasticity } \\
\hline 1 & 0.05 & 0.05 & 0.05 & 0.05 & 0.05 \\
\hline 2 & 0.05 & 0.05 & 0.05 & 0.05 & 0.05 \\
\hline 3 & 0.04 & 0.04 & 0.04 & 0.02 & 0.02 \\
\hline \multicolumn{6}{|c|}{ Model 2: Periodic Heteroscedasticity } \\
\hline 1 & 0.06 & 0.05 & 0.05 & 0.05 & 0.05 \\
\hline 2 & 0.05 & 0.05 & 0.05 & 0.06 & 0.06 \\
\hline 3 & 0.04 & 0.04 & 0.04 & 0.03 & 0.03 \\
\hline \multicolumn{6}{|c|}{ Model 3: Single Volatility Break } \\
\hline 1 & 0.06 & 0.05 & 0.05 & 0.05 & 0.05 \\
\hline 2 & 0.05 & 0.05 & 0.05 & 0.05 & 0.05 \\
\hline 3 & 0.03 & 0.04 & 0.03 & 0.03 & 0.03 \\
\hline \multicolumn{6}{|c|}{ Model 4: Single Periodic Volatility Shift } \\
\hline 1 & 0.05 & 0.05 & 0.05 & 0.05 & 0.05 \\
\hline 2 & 0.05 & 0.05 & 0.05 & 0.05 & 0.05 \\
\hline 3 & 0.04 & 0.03 & 0.03 & 0.02 & 0.03 \\
\hline \multicolumn{6}{|c|}{ Model 5: Trending Volatility } \\
\hline 1 & 0.05 & 0.05 & 0.05 & 0.05 & 0.05 \\
\hline 2 & 0.05 & 0.05 & 0.05 & 0.05 & 0.05 \\
\hline 3 & 0.04 & 0.03 & 0.03 & 0.02 & 0.03 \\
\hline \multicolumn{6}{|c|}{ Model 6: Exponential Stochastic Integrated Volatility } \\
\hline 1 & 0.05 & 0.05 & 0.05 & 0.05 & 0.05 \\
\hline 2 & 0.05 & 0.05 & 0.05 & 0.05 & 0.05 \\
\hline 3 & 0.04 & 0.05 & 0.04 & 0.03 & 0.03 \\
\hline
\end{tabular}


Table 15 Size-adjusted power of wild bootstrap FSVR seasonal unit root tests for $S=12$ : GLS demeaning

\begin{tabular}{lllllll}
\hline Serial Correlation & $c$ & $\tau_{0}^{*}$ & $\tau_{2}^{*}$ & $\tau_{1}^{*}$ & $\tau_{012}^{*}$ & $\tau_{1}^{*}$ \\
\hline $\begin{array}{l}\text { Model 1: Homoscedasticity } \\
\text { i.i.d. }\end{array}$ & & & & & \\
& 13.5 & 0.67 & 0.66 & 0.96 & 1 & 1 \\
2 & 7 & 0.34 & 0.31 & 0.64 & 1 & 1 \\
2 & 13.5 & 0.67 & 0.64 & 0.96 & 1 & 1 \\
3 & 7 & 0.27 & 0.25 & 0.54 & 1 & 1 \\
3 & 13.5 & 0.59 & 0.59 & 0.92 & 1 & 1
\end{tabular}

Model 2: Periodic Heteroscedasticity

$\begin{array}{lllllll}1 & 7 & 0.33 & 0.32 & 0.63 & 1 & 1 \\ 1 & 13.5 & 0.67 & 0.65 & 0.95 & 1 & 1 \\ 2 & 7 & 0.34 & 0.33 & 0.63 & 1 & 1 \\ 2 & 13.5 & 0.68 & 0.66 & 0.96 & 1 & 1 \\ 3 & 7 & 0.26 & 0.25 & 0.54 & 1 & 0.99 \\ 3 & 13.5 & 0.58 & 0.58 & 0.93 & 1 & 1 \\ \text { Model 3: Single Volatility } \text { Break } & & & & & \\ 1 & 7 & 0.34 & 0.34 & 0.36 & 0.38 & 0.38 \\ 1 & 13.5 & 0.67 & 0.67 & 0.7 & 0.71 & 0.71 \\ 2 & 7 & 0.34 & 0.33 & 0.36 & 0.37 & 0.37 \\ 2 & 13.5 & 0.68 & 0.67 & 0.7 & 0.71 & 0.71 \\ 3 & 7 & 0.28 & 0.29 & 0.3 & 0.31 & 0.3 \\ 3 & 13.5 & 0.62 & 0.62 & 0.64 & 0.65 & 0.65\end{array}$

Model 4: Single Periodic Volatility Shift

\begin{tabular}{|c|c|c|c|c|c|c|}
\hline 1 & 7 & 0.25 & 0.24 & 0.52 & 1 & 1 \\
\hline 1 & 13.5 & 0.47 & 0.47 & 0.82 & 1 & 1 \\
\hline 2 & 7 & 0.24 & 0.25 & 0.5 & 1 & 1 \\
\hline 2 & 13.5 & 0.47 & 0.46 & 0.8 & 1 & 1 \\
\hline 3 & 7 & 0.23 & 0.22 & 0.48 & 1 & 1 \\
\hline 3 & 13.5 & 0.46 & 0.45 & 0.85 & 1 & 1 \\
\hline \multicolumn{7}{|c|}{ Model 5: Trending Volatility } \\
\hline 1 & 7 & 0.31 & 0.32 & 0.62 & 1 & 1 \\
\hline 1 & 13.5 & 0.62 & 0.63 & 0.94 & 1 & 1 \\
\hline 2 & 7 & 0.32 & 0.31 & 0.64 & 1 & 1 \\
\hline 2 & 13.5 & 0.63 & 0.64 & 0.94 & 1 & 1 \\
\hline 3 & 7 & 0.27 & 0.26 & 0.55 & 1 & 1 \\
\hline 3 & 13.5 & 0.59 & 0.57 & 0.93 & 1 & 1 \\
\hline \multicolumn{7}{|c|}{ Model 6: Exponential Stochastic Integrated Volatility } \\
\hline 1 & 7 & 0.32 & 0.32 & 0.64 & 1 & 1 \\
\hline 1 & 13.5 & 0.61 & 0.62 & 0.93 & 1 & 1 \\
\hline
\end{tabular}


Table 15 continued

\begin{tabular}{lllllll}
\hline Serial Correlation & $c$ & $\tau_{0}^{*}$ & $\tau_{2}^{*}$ & $\tau_{1}^{*}$ & $\tau_{012}^{*}$ & $\tau_{12}^{*}$ \\
\hline 2 & 7 & 0.31 & 0.31 & 0.61 & 1 & 1 \\
2 & 13.5 & 0.6 & 0.61 & 0.92 & 1 & 1 \\
3 & 7 & 0.26 & 0.3 & 0.6 & 1 & 1 \\
3 & 13.5 & 0.57 & 0.61 & 0.94 & 1 & 1 \\
\hline
\end{tabular}

\section{References}

Basawa IV, Mallik AK, McCormick WP, Reeves JH, Taylor RL (1991) Bootstrapping unstable first-order autoregressive processes. Annals Stat 19(2):1098-1101

Beaulieu JJ, Miron JA (1993) Seasonal unit roots in aggregate us data. J Econ 55(1-2):305-328

Boswijk HP, Cavaliere G, Rahbek A, Taylor AR (2016) Inference on co-integration parameters in heteroskedastic vector autoregressions. J Econ 192(1):64-85

Burridge P, Taylor AR (2001) On regression-based tests for seasonal unit roots in the presence of periodic heteroscedasticity. J Econ 104(1):91-117

Burridge P, Taylor AR (2004) Bootstrapping the hegy seasonal unit root tests. J Econ 123(1):67-87

Cavaliere G, Skrobotov A, Taylor AR (2017) Wild bootstrap seasonal unit root tests for time series with periodic nonstationary volatility. Econometric Reviews, pp. 1-24

Cavaliere G, Taylor AR (2007) Testing for unit roots in time series models with non-stationary volatility. J Econ 140(2):919-947

Cavaliere G, Taylor AR (2008) Bootstrap unit root tests for time series with nonstationary volatility. Econ Theory 24(1):43-71

Cavaliere G, Taylor AR (2009) Heteroskedastic time series with a unit root. Econ Theory 25(5):1228-1276

del Barrio Castro T, Osborn DR, Taylor AR (2012) On augmented hegy tests for seasonal unit roots. Econ Theory 28(5):1121-1143

del Barrio Castro T, Rodrigues PM, Taylor AR (2018) Semi-parametric seasonal unit root tests. Econ Theory 34(2):447-476

Eroğlu BA, Göğebakan KÇ, Trokić M (2018) Powerful nonparametric seasonal unit root tests. Econ Letts 167:75-80

Eroğlu BA, Yiğit T (2016) A nonparametric unit root test under nonstationary volatility. Econ Letts 140:6-10

Franses PH et al. (1996) Periodicity and stochastic trends in economic time series. OUP Catalogue

Hansen BE (2000) Testing for structural change in conditional models. J Econ 97(1):93-115

Hylleberg S, Engle RF, Granger CW, Yoo BS (1990) Seasonal integration and cointegration. J Econ 44(12):215-238

Kahn JA, McConnell MM, Perez-Quiros G et al (2002) On the causes of the increased stability of the us economy. Federal Reserve Bank New York Economic Policy Review 8(1):183-202

McConnell MM, Perez-Quiros G (2000) Output fluctuations in the united states: What has changed since the early 1980's? Am Econ Rev 90(5):1464-1476

Nielsen MØ (2010) Nonparametric cointegration analysis of fractional systems with unknown integration orders. J Econ 155(2):170-187

Proietti T (1998) Seasonal heteroscedasticity and trends. J Forecast 17(1):1-17

Smith RJ, Taylor AR, del Barrio Castro T (2009) Regression-based seasonal unit root tests. Econ Theory 25(2):527-560

Van Dijk D, Osborn D, Sensier M (2002) Changes in variability of the business cycle in the g7 countries. Technical Report

Publisher's Note Springer Nature remains neutral with regard to jurisdictional claims in published maps and institutional affiliations. 